\title{
ON THE PROJECTIVE DIMENSION OF 5 QUADRIC ALMOST COMPLETE INTERSECTIONS WITH LOW MULTIPLICITIES
}

\author{
SABINE EL KHOURY
}

\begin{abstract}
Let $S$ be a polynomial ring over an algebraic closed field $k$ and $\mathfrak{p}=(x, y, z, w)$ a homogeneous height four prime ideal. We give a finite characterization of the degree two component of ideals primary to $\mathfrak{p}$, with multiplicity $e \leq 3$. We use this result to give a tight bound on the projective dimension of almost complete intersections generated by five quadrics with $e \leq 3$.
\end{abstract}

\section{INTRODUCTION}

Let $S$ be the polynomial ring over an algebraically closed field $k$, and $x, y, z, w \in S$ four linearly independent linear forms. When $\mathfrak{p}=(x, y)$ is a height 2 prime ideal, Engheta shows there are two distinct types of p-primary ideals of multiplicity 2, [6, Proposition 11]. As a consequence, he proves that if $I$ is an ideal generated by three cubics, then the projective dimension of $S / I$ is at most 36 [7]. This answers a specific case to Stillman's question:

Question 1.1. (Stillman [21, Problem 3.14]) Is there a bound on the pd(S/I) depending only on $d_{1}, \ldots, d_{N}$ and $N$, where $d_{i}=\operatorname{deg}\left(f_{i}\right)$ ?

Furthermore, the bound found by Engheta is not optimal see [8] and [19], as the expected projective dimension of three cubics is 5. In [12], Huneke et.al. showed that for each $e \geq 3$ and for any $n \in \mathbb{N}$, there exists an ideal $J$ primary to a linear prime ideal $\left(x_{1}, \ldots x_{h}\right)$, with $e(S / J)=e$ and $\operatorname{pd}(S / J) \geq n$. Therefore, no natural extensions for Engheta's result on multiplicity 2 primary ideals would be possible. In [17], Mantero and McCullough found a finite classification for p-primary ideals $J$ of multiplicities 3 and 4 , by imposing an upper bound on the degrees of the generators of $J$. In particular, they find a finite classification of the linear, quadric and cubic generators of such p-primary ideals of multiplicities 3 and 4 . They use their results to improve Engheta's estimates on three cubics, and show that the projective dimension is at most 5, [18]. Stillman's question was proven recently by Ananyan and Hochster [1]. They show the existence of a bound for any homogenous ideal $I$ generated by $N$ forms of degree at most $d$. However, the bounds they produce are very large even for ideals generated by quadrics or cubics. In a previous paper [2], they get a tighter bound for ideals generated by $N$ quadrics, but again not optimal. It remains open to find what are the best bounds for projective dimensions.

When $\mathfrak{p}=(x, y, z)$, Huneke et.al. give a characterization of the degree 2 component of an ideal $J$ primary to $\mathfrak{p}$ with $\mathrm{e}(S / J) \leq 4$, see [11]. By using this classification, they obtain a tight upper bound for the projective dimension generated by four quadrics. They show that if $I$ is generated by four quadrics, then $\operatorname{pd}(S / I) \leq 6$. They also pose the following question:

Question 1.2. ([11, Question 10.2] and [13, Question 6.2]) Let $S$ be a polynomial ring, and let I be an ideal of $S$ generated by $n$ quadrics and having $h t(I)=h$. Is it true that $p d(S / I) \leq h(n-h+1)$ ?

In our paper, we treat the case when $\mathfrak{p}=(x, y, z, w)$. Our results, Propositions 3.4 and 4.4 , offer a finite classification, in the sense of [11], for $\mathfrak{p}$-primary ideals $J$ with multiplicity $e(S / J) \leq 3$. Our characterization depends on the degree two component of $J$. We use these classifications along with a result of [3], to answer Question 1.2 for almost complete intersections $I$ generated by 5 quadrics with low multiplicities. We show

Date: $2,19,2018$.

1991 Mathematics Subject Classification. 13D02; 13D05.

Key words and phrases. projective dimension, almost complete intersections, primary ideals. 
that if $I$ is an almost complete intersection with multiplicity $e(S / I) \leq 3$, then $\operatorname{pd}(S / I) \leq 8$.

Beyond the applications to Stillman's question, different characterization and structures of $\mathfrak{p}$-primary ideals have been given by algebraic geometers who study vector bundles and multiple or nilpotent structures, see [15], [16] and [24] for instance.

The rest of the paper is divided in the following way. In section 2 , we collect results that are useful to us and set our notation. In Sections 3 and 4, we give our characterization of height four primary ideals with multiplicities 2 and 3 respectively. These primary ideals can occur as components of the unmixed part of our 5 quadrics ideal or a direct link to this ideal. In section 4 , we apply our previous results to prove that the projective dimension of an almost complete intersection generated by 5 quadrics with $e \leq 3$ is at most 8. Appendix $A$ contains a list of primary, unmixed ideals that are essential to our sections.

\section{Preliminaries}

In this section, we set our notations and collect results that are useful to our theorems.

Unmixed ideals and multiplicity. We use the associativity formula to compute the multiplicity of an ideal

Proposition 2.1 (Associativity Formula). [14, Theorem 11.2.4] If $J$ is an ideal of S, then

$$
e(S / J)=\sum_{\substack{J \subset \mathfrak{p} \\ h t(\mathfrak{p})=h t(J)}} e(S / \mathfrak{p}) \lambda\left(S_{\mathfrak{p}} / J_{\mathfrak{p}}\right)
$$

where $e(S)$ denotes the multiplicity of a graded ring $S$, and $\lambda(M)$ denotes the length of an $S$-module $M$.

An ideal $J$ of height $h$ is unmixed if $\operatorname{ht}(\mathfrak{p})=h$ for every $\mathfrak{p} \in \operatorname{Ass}(S / J)$. The unmixed part of $J$, denoted $J^{u n}$, is the intersection of all the components of $\mathrm{J}$ of minimum height. We have $J \subset J^{u n}$, and $e(S / J)=e\left(S / J^{u n}\right)$. We follow the notation of [6]: if $J$ is an unmixed ideal, we say it is of type $\left\langle e_{1}, \ldots, e_{m} ; \lambda_{1}, \ldots, \lambda_{m}\right\rangle$. If $J$ has $m$ associated prime ideals $\mathfrak{p}_{1}, \mathfrak{p}_{2} \ldots \mathfrak{p}_{m}$, then $e(S / J)=\sum_{i=1}^{m} e_{i} \lambda_{i}$, where $e_{i}=e(S / \mathfrak{p})$ and $\lambda_{i}=\lambda\left(S_{\mathfrak{p}_{i}} / J_{\mathfrak{p}_{i}}\right)$. We also get

Lemma 2.2. [6, lemma 8] Let $J \subset S$ be an unmixed ideal. If $I \subset S$ is an ideal containing $J$ such that $h t(I)=h t(J)$ and $e(S / I)=e(S / J)$, then $J=I$.

Linkage. Two ideals $J$ and $K$ in a regular $\operatorname{ring} R$ are said to be linked $J \sim K$, if there exists a regular sequence $\underline{\alpha}=\alpha_{1}, \ldots \alpha_{g}$ such that $K=(\underline{\alpha}): J$ and $J=(\underline{\alpha}): K$. Notice that the definition forces $J$ and $K$ to be unmixed, and $(\underline{\alpha}) \subset J \cap K$.

The following results on linkage are needed.

Theorem 2.3. (Peskine-Szpiro [23]) Let $J$ be an unmixed ideal of $S$ of height $g$. Let $\underline{\alpha}=\alpha_{1}, \ldots, \alpha_{g}$ be a regular sequence in $J$, and set $K=(\underline{\alpha}): J$. Then one has

(i) $J=(\underline{\alpha}): K$, that is, $J \sim K$ via $\underline{\alpha}$;

(ii) $S / J$ is $C M$ if and only if $S / K$ is $C M$;

(iii) $e(S / J)+e(S / K)=e(S /(\underline{\alpha}))$

If $(\underline{\alpha})$ is a regular sequence of maximal length in an ideal $I$, then $(\underline{\alpha}): I=(\underline{\alpha}): I^{u n}$ that is $I^{u n}$ is linked to $(\underline{\alpha}): I$.

Lemma 2.4. [5, Lemma 2.6] Let $R$ be a Gorenstein local ring and let $I \subset R$ be an unmixed ideal. All ideals which are linked to $I$ have the same (finite or infinite) projective dimension.

Lemma 2.5. [6, Theorem 7] Let $J$ be an almost complete intersection ideal of $S$. If $K$ is an ideal linked to $J^{u n}$, then $\operatorname{pd}(S / J) \leq p d(S / K)+1$ 
ON THE PROJECTIVE DIMENSION OF 5 QUADRIC ALMOST COMPLETE INTERSECTIONS WITH LOW MULTIPLICITIES3

\section{Basic Results.}

Theorem 2.6. (Samuel [22], Nagata [20, Theorem 40.6]) Let J be a homogeneous unmixed ideal of S. If $e(S / J)=1$, then $J$ is generated by $h t(J)$ linear forms.

A homogeneous ideal $I$ is called degenerate if $I$ contains at least one linear form, otherwise $I$ is said to be non-degenerate. We need the next result on a lower bound for the multiplicity of non-degenerate prime ideals.

Proposition 2.7. [10, Corollary 18.12] Let $\mathfrak{p}$ be a homogeneous prime ideal of $S$. If $\mathfrak{p}$ is a non-degenerate prime ideal, then $e(S / \mathfrak{p}) \geq h t(\mathfrak{p})+1$

Corollary 2.8. Let $\mathfrak{p}$ be a homogeneous prime ideal of $S$ of height four. If $e(S / \mathfrak{p})=2$, then there exists linear forms $x, y, z$ and a quadric $q$ such that $\mathfrak{p}=(x, y, z, q)$

The following consequence of a result of Ananyan and Hochster [2, Lemma 3.3] is essential to our paper.

Lemma 2.9. [2, Lemma 3.3] Let $f_{1} \ldots f_{t}$ be a regular sequence of forms in $S$ and set $A=k\left[f_{1}, \ldots, f_{t}\right]$. Then for any ideal $I$ of $S$ extended from $A$, one has $p d(S / I) \leq t$. More generally for any finite $S$-module $M$ presented by a matrix with entries in $A$, one has $p d(M) \leq t$. In particular, any ideal $I$ whose generators can be written in terms of at most $t$ variables satisfies $p d(S / I) \leq t$.

The following Corollary of Lemma 2.9 applies to almost complete intersections.

Corollary 2.10. [11, Corollary 3.9] Let I be an almost complete intersection of height h. Suppose $I^{\text {un }}$ is extended from $A=k\left[f_{1}, \ldots, f_{t}\right]$ where $f_{1}, \ldots f_{t}$ form a regular sequence then $p d(S / I) \leq \max (h+2, t)$

Proposition 2.11. [11, Proposition 3.7] Let I be an almost complete intersection of height h. Let $F_{\bullet}$ be the minimal free resolution of the unmixed part $I^{\text {un }}$ of $I$. Let $\partial_{i}$ denote the $i^{\text {th }}$ differential in $F_{\bullet}$. Then

$$
\operatorname{pd}(S / I) \leq \max \left\{h+2, \operatorname{pd}\left(\operatorname{Coker}\left(\partial_{h+1}^{*}\right)\right)\right.
$$

Lemma 2.12. [11, Lemma 3.6] Suppose $I_{1}, I_{2}$ are two ideals. Then

$$
p d\left(S /\left(I_{1} \cap I_{2}\right)\right) \leq \max \left\{p d\left(S / I_{1}\right), p d\left(S / I_{2}\right), p d\left(S /\left(I_{1}+I_{2}\right)-1\right)\right\}
$$

\section{3. $(x, y, z, w)$-PRIMARY OF MULTIPLICITY 2}

In this section, we give a characterization of the degree 2 component of an ideal primary to the linear prime $\mathfrak{p}=(x, y, z, w)$ of multiplicity 2 , similar to the characterization found by C. Huneke et al. [11, Proposition 4.3]. Before we give our main result, we prove lemmas on matrices of linear forms that are essential to the characterization of these ideals. For any $m \times n$ matrix $M$ with $(m \leq n)$, we denote $I_{j}(M)$ to be the ideal generated by the $j \times j$ minors of $M . I_{j}(M)$ is unchanged by linear row and column operations. A matrix $M$ is 1-generic if it does not have a zero entry called a generalized zero, after row/column operations. If $M$ is 1 - generic, then the ideal $I_{m}(M)$ generated by the maximal minors of $M$ is prime and of codimension $n-m+1,[4$, Theorem 6.4].

Lemma 3.1. Let $M=\left(\begin{array}{cccc}a & b & c & d \\ e & f & g & h\end{array}\right)$ where $a, \ldots h$ are linear forms, such that $h t\left(I_{2}(M)\right)=1$. Then, after row and column operations, $M$ is one of the following

(i) $M=\left(\begin{array}{llll}a & 0 & 0 & 0 \\ e & f & g & h\end{array}\right)$ with $a \neq 0$.

(ii) $M=\left(\begin{array}{llll}a & b & 0 & 0 \\ e & f & 0 & 0\end{array}\right)$ with $a f-b e \neq 0$.

(iii) $M=\left(\begin{array}{llll}a & b & 0 & 0 \\ e & 0 & b & 0\end{array}\right)$ with ht $(a, b, e)=3$. 
Proof. Since ht $\left(I_{2}(M)\right)=1$ then $M$ is not 1-generic [4, Theorem 6.4] and has a generalized zero, say $d=0$.

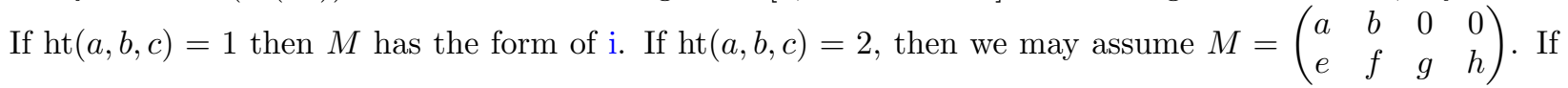
ht $(e, f, g, h) \geq 3$ then, since $I_{2}(M)=(a f-b e, a g, a h, b g, b h)=(a, b) \cap(a f-b e, g, h)$, we get ht $\left(I_{2}(M)\right)>1$. Hence, we may assume ht $(e, f, g, h)=2$ (when it is equal to 1 , we $M$ has the form of i). So the only two possibilities for $M$ would be

$$
M=\left(\begin{array}{cccc}
a & b & 0 & 0 \\
e & f & 0 & 0
\end{array}\right) \quad \text { or } \quad M=\left(\begin{array}{cccc}
a & b & 0 & 0 \\
e & 0 & f & 0
\end{array}\right)
$$

In the latter case, we have $I_{2}(M)=(b e, a f, b f)$ which implies $f \in(b)$. This puts us in case iii.

Finally, when $\operatorname{ht}(a, b, c)=3$, then $I_{2}(M)=(a f-b e, a g-c e, b g-c f, a h, b h, c h)=(a f-b e, a g-c e, b g-c f, h) \cap$ $(a, b, c)$. This implies that ht $\left(I_{2}\left(\begin{array}{lll}a & b & c \\ e & f & g\end{array}\right), h\right)=1$. Hence, by [11, Lemma 4.1], we get ht $(e, f, g, h)=1$, which puts us in i.

Lemma 3.2. Let $M=\left(\begin{array}{cccc}a & b & c & d \\ e & f & g & h \\ k & l & m & n\end{array}\right)$ be a $3 \times 4$ matrix of generic forms. Suppose ht $\left(I_{3}(M)\right)=1$ then, after row and column operations, $M$ has one of the following form

(i) $M=\left(\begin{array}{cccc}a & 0 & 0 & 0 \\ e & f & g & h \\ k & l & m & n\end{array}\right)$ with $a \neq 0$ and $h t\left(I_{2}\left(\begin{array}{ccc}f & g & h \\ l & m & n\end{array}\right)\right)>0$.

(ii) $M=\left(\begin{array}{llcc}a & b & 0 & 0 \\ e & f & 0 & 0 \\ k & l & m & n\end{array}\right)$ with $a f-b e \neq 0$ and $h t(m, n)>0$.

(iii) $M=\left(\begin{array}{cccc}a & b & 0 & 0 \\ e & 0 & b & 0 \\ k & l & m & n\end{array}\right)$ with $n \neq 0$ and $h t(a, b, e)=3$.

(iv) $M=\left(\begin{array}{llll}a & b & 0 & 0 \\ e & f & n & 0 \\ k & l & 0 & n\end{array}\right)$ with $n \neq 0$ and $h t(a, b)=2$.

(v) $M=\left(\begin{array}{cccc}a & b & c & 0 \\ e & f & g & 0 \\ k & l & m & 0\end{array}\right)$ with $\operatorname{det}\left(\begin{array}{ccc}a & b & c \\ e & f & g \\ k & l & m\end{array}\right) \neq 0$.

(vi) The form of $M$ is determined by at most four variables.

Proof. Since ht $\left(I_{3}(M)\right)=1$ then $M$ is not 1- generic [4, Theorem 6.4] and has a generalized zero. So we may assume that $M$ has the form of $M=\left(\begin{array}{cccc}a & b & c & 0 \\ e & f & g & h \\ k & l & m & n\end{array}\right)$. If $\operatorname{ht}(a, b, c)=1$, then $M$ has the form of i. Suppose $\operatorname{ht}(a, b, c) \geq 2$ and consider $M_{1}=\left(\begin{array}{cccc}e & f & g & h \\ k & l & m & n\end{array}\right) . M_{1}$ is not 1-generic and has a generalized zero, or else ht $\left(I_{3}(M)\right) \neq 1$. Hence, after a column operation and a linear change of variables, $M$ is equal to either $M=\left(\begin{array}{cccc}a & b & c & 0 \\ e & f & g & 0 \\ k & l & m & n\end{array}\right)$ or $M=\left(\begin{array}{cccc}a & b & c & 0 \\ e & f & 0 & h \\ k & l & m & n\end{array}\right)$. So for the rest of the proof, we assume ht $(e, f, g, h) \leq 3$.

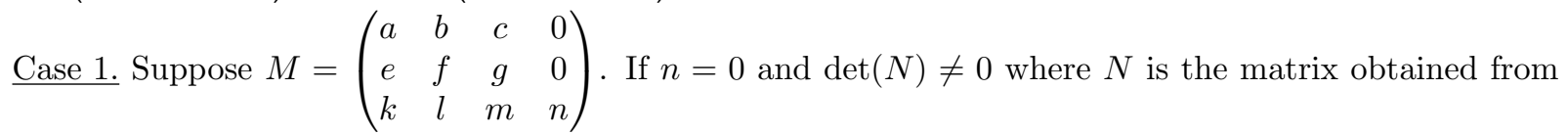
$M$ by deleting the last column, then $M$ has the form of $\mathrm{v}$. We assume $n \neq 0$, and we write $I_{3}(M)=$ $\left(\operatorname{det} N, n \delta_{1}, n \delta_{2}, n \delta_{3}\right)$ where $\left(\delta_{1}, \delta_{2}, \delta_{3}\right)=I_{2}\left(M^{\prime}\right)=I_{2}\left(\begin{array}{lll}a & b & c \\ e & f & g\end{array}\right)$. Hence $I_{3}(M)=\left(\delta_{1}, \delta_{2}, \delta_{3}\right) \cap(n, \operatorname{det} N)$. 
ON THE PROJECTIVE DIMENSION OF 5 QUADRIC ALMOST COMPLETE INTERSECTIONS WITH LOW MULTIPLICITIES5

We also write $\operatorname{det}(N)=k(b g-f c)-l(a g-e c)+m(a f-b e)=k \delta_{1}-l \delta_{2}+m \delta_{3}$. Since ht $\left(I_{3}(M)\right)=1$, then either $\operatorname{ht}\left(\delta_{1}, \delta_{2}, \delta_{3}\right)=\operatorname{ht}\left(I_{2}\left(M^{\prime}\right)\right)=1 \operatorname{or} \operatorname{det} N \in(n)$ (when $\operatorname{det}(N) \in k, l$ or $m$, we get back to one of the two cases). If $\operatorname{ht}\left(I_{2}\left(M^{\prime}\right)\right)=1$ then by [11, Lemma 4.1], we get either i, ii, or iii. If $\operatorname{det}(N) \in(n)$, then we get either $\delta_{1}, \delta_{2}, \delta_{3} \in(n)$ or $k, l, m \in(n)$ or $\delta_{1}, \delta_{2}, m \in(n)$ or $\delta_{1}, l, m \in(n)$, and the remaining cases are identical. The first case implies ht $\left(I_{2}\left(M^{\prime}\right)\right)=1$, which was treated above. The second case puts us in i, the third one in either i or iv, and the last one in either iii or iv.

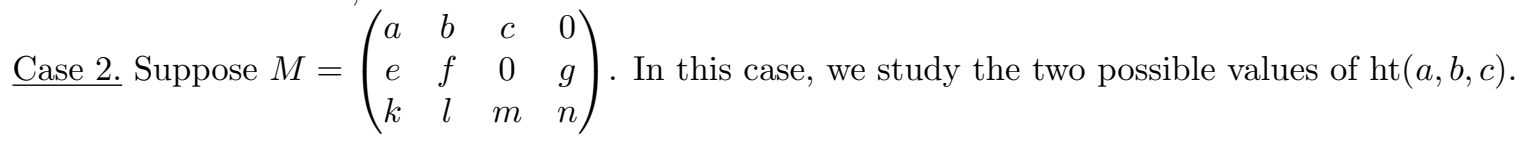

(i) Suppose $\operatorname{ht}(a, b, c)=2$, then either $M=\left(\begin{array}{cccc}a & b & 0 & 0 \\ e & f & g & 0 \\ k & l & m & n\end{array}\right)$ or $M=\left(\begin{array}{cccc}a & b & 0 & 0 \\ e & 0 & f & g \\ k & l & m & n\end{array}\right)$. The former case was already treated in Case 1 , so we discuss the latter case.

- If ht $(e, f, g) \leq 1$ then we are back to case i.

- If ht $(e, f, g)=2$, then $M$ have one of the following forms:

$$
\text { a) } \left.M=\left(\begin{array}{cccc}
a & b & 0 & 0 \\
0 & e & f & 0 \\
k & l & m & n
\end{array}\right) \text { or } \quad b\right) M=\left(\begin{array}{cccc}
a & b & 0 & 0 \\
0 & 0 & e & f \\
k & l & m & n
\end{array}\right) \quad \text { with } \operatorname{ht}(a, b)=\operatorname{ht}(e, f)=2 \text {. }
$$

- If $M$ has the form of $a$ ), then we get back to Case 1.

- Suppose $M$ has the form of $b)$. We have $I_{3}(M)=(e(a l-b k), f(a l-b k), a(e n-f m), b(e n-f m))=$ $(a, b) \cap(e, f) \cap(a l-b k, e n-f m)$ with height equal to 1 . We only study the cases when $I_{3}(M) \subset(a)$ or $I_{3}(M) \subset(a l-b k)$, since the other ones are obtained in a similar manner after a linear change of variables. If $I_{3}(M) \subset(a)$, then $e b k, f b k$, ben, bf $m \in(a)$. This implies that $k \in(a)$, and either $e, m \in(a)$ or $m, n \in(a)$. We may assume $k=0$ after a row operation. If $e, m \in(a)$, then we get back to iv. If $m, n \in(a)$, then we get back to iii after row/column operations and a linear change of variables. When $I_{3}(M)=(e(a l-b k), f(a l-b k), a(f m-e n), b(f m-e n)) \subset(a l-b k)$, then $(e n-f m) \in(a l-b k)$ and the form of $M$ is defined by 4 variables which puts us in vi.

- Suppose ht $(e, f, g)=3$ and write $M=\left(\begin{array}{cccc}a & b & 0 & 0 \\ e & 0 & f & g \\ k & l & m & n\end{array}\right)$. We have $I_{3}(M)=(a f l-b(e m-$ $f k), a g l-b(e n-g k), a(f n-g m), b(f n-g m))=(a, b) \cap(f n-g m, a f l-b(e m-f k), a g l-b(e n-g k))$ with height 1 . We may assume that $\operatorname{ht}(k, l, m, n) \geq 3$, since otherwise this would be identical to the case when $\operatorname{ht}(e, f, g)=2$. If $I_{3}(M) \subset(a)$, then $(e m-f k),(e n-g k),(f n-g m) \in(a)$. This implies that ht $\left(I_{2}\left(\begin{array}{ccc}e & f & g \\ k & m & n\end{array}\right)\right)=1$. By [11, Lemma 4.1], we get either ht $(e, f, g) \leq 2$ or $\operatorname{ht}(k, m, n)=1$, which is impossible. If $I_{3}(M) \subset(b)$, then $l, f n-g m \in(b)$. We may assume $l=0$ after a row operation and we are back to Case 1. If $I_{3}(M) \not \subset(a)$ nor in $(b)$, then $\operatorname{ht}\left((f n-g m, a f l-b(e m-f k), a g l-b(e n-g k))=1\right.$. We get ht $\left(I_{2}\left(\begin{array}{ccc}e & f & g \\ k & m & n\end{array}\right), f l, g l\right)=1$. Again, by [11, Lemma 4.1], we get ht $(e, f, g) \leq 2$ or ht $(k, m, n)=1$ which is impossible.

(ii) Suppose $\operatorname{ht}(a, b, c)=3$, and $M=\left(\begin{array}{cccc}a & b & c & 0 \\ e & f & 0 & g \\ k & l & m & n\end{array}\right)$. Again, we assume that ht $(e, f, g)=3$ and $\operatorname{ht}(k, l, m, n) \geq 3$, since otherwise these cases would be identical to whenever $\operatorname{ht}(a, b, c) \leq 2$. We have $I_{3}(M)=(a f m-b e m+c(e l-f k), a(f n-g l)-b(e n-g k), a g m+c(e n-g k), b g m+c(f n-g l))$. Since $\operatorname{ht}\left(I_{3}(M)\right)=1$, then $I_{3}(M)$ is contained in a prime ideal $P$ of height 1 . Every prime ideal of height 1 in a UFD is principal, then $P$ is generated by a either a degree 1 , a degree 2 or a degree three element. If $P$ is generated by a degree one element $u$, then $u$ would be one of the variables $a, b, c, e, f, g, k, l, m$ or $n$. The cases when $I_{3}(M) \subset(a),(b),(e)$ or $(f)$ are identical, so we treat 
one of them. If $I_{3}(M) \subset(a)$, then $e m, g m,(e l-f k),(e n-g k),(f n-g l) \in(a)$. It implies that $\mathrm{ht}\left(I_{2}\left(\begin{array}{lll}e & f & g \\ k & l & n\end{array}\right), e m, g m\right)=1$. By [11, Lemma 4.1], we get ht $(e, f, g) \leq 2$ or ht $(k, m, n)=1$ which is impossible. Again the cases of $I_{3}(M) \subset(c)$ or $(g)$ are identical. If $I_{3}(M) \subset(c)$, then $f m, e m, g m,(f n-g l),(e n-g k) \in(c)$. Hence either $m, n, g \in(c)$ and $M$ has the form of iv, or $k, l, m \in(n)$ which is impossible in this case (we actually get back to i but we assumed that $h t(k, l, m, n) \geq 3)$. If $I_{3}(M) \subset(k),(l),(m)$ or $(n)$, then we again get back to the case when $\mathrm{ht}(a, b, c) \leq 2$. Suppose $I_{3}(M)$ is contained in a prime ideal generated by a degree two form $u$. Since every term in $I_{3}(M)$ is a product of three linear forms of $M$, we may assume $u \in I_{2}(M)$. We either get ht $\left(I_{2}\left(\begin{array}{cccc}e & f & 0 & g \\ k & l & m & n\end{array}\right)\right)=1$ or ht $\left(I_{2}\left(\begin{array}{cccc}a & b & c & 0 \\ k & l & m & n\end{array}\right)\right)=1$ which are both impossible by Lemma 3.1, or we obtain cases that lead to either ht $(e, f, g)$ or ht $(k, l, m, n)$ less than 3 which is also a contradiction. Finally, if $u$ is a form of degree three, then $u$ must be one of the generators of $I_{3}(M)$. We again get to ht $(e, f, g) \leq 3$ or ht $(k, l, m, n) \leq 3$.

Remark 3.3. We record the matrices of 3.2-vi as details are needed to complete the proof of Proposition 3.4:

(i) $M=\left(\begin{array}{llll}a & b & 0 & 0 \\ 0 & 0 & k & l \\ k & l & a & b\end{array}\right)$ with ht $(a l-b k)=1$.
(ii) $M=\left(\begin{array}{llll}a & b & 0 & 0 \\ 0 & 0 & k & a \\ k & l & l & b\end{array}\right)$ with ht $(a l-b k)=1$.

We then get to our first result on the characterization of $(x, y, z, w)$-primary ideals.

Proposition 3.4. Let $J$ be an $(x, y, z, w)$-primary with $e(S / J)=2$ then one of the following holds:

I- If $J$ is degenerate then

(i) $J=\left(x, y, z, w^{2}\right)$.

(ii) $J=(x, y)+(z, w)^{2}+(a z+b w), h t(x, y, z, w, a, b)=6$.

(iii) $J=(x)+(y, z, w)^{2}+(b y+c z+d w, e y+f z+g w)$ with $h t\left(x, y, z, w, I_{2}\left(\begin{array}{lll}b & c & d \\ e & f & g\end{array}\right)\right) \geq 6$.

(iv) All quadrics in $J$ can be written in terms of at most of 8 variables.

II- If $J$ is non-degenerate then

(i) $J=(x, y, z, w)^{2}+(a x+b y+c z+d w, e x+f y+g z+h w, k x+l y+m z+n w)$ with $h t\left(x, y, z, w, I_{3}(M) \geq\right.$ 6 , where $M=\left(\begin{array}{cccc}a & b & c & d \\ e & f & g & h \\ k & l & m & n\end{array}\right)$.

(ii) $J=(x, y, z, w)^{2}+(a x+b y, e x+f y+n z, k x+l y+n w,(a f-b e) w-(a l-k b) z)$ with $h t(a, b)=2$ and $n \neq 0$.

(iii) $J=(x, y, z, w)^{2}+(a x+b y, e x+b z, e y-a z, k x+l y+m z+n w)$ with $h t(a, b, e)=3$ and $n \neq 0$.

(iv) All quadrics in $J$ can be written in terms of at most of 8 variables.

Proof. The proof of the first three cases goes along the same line as the first cases of [11, Proposition 4.3]. Since $e(S / J)=2$, we have $(x, y, z, w)^{2} \subset J \subset(x, y, z, w)$. If $J$ contains a linear form, then $J$ can be written as $J=(x)+\left(J^{\prime}\right)$ where $J^{\prime}$ is $(y, z, w)$-primary with multiplicity 2. By [11, Proposition 4.3], the authors obtained 4 different cases for $J^{\prime}$, and hence $J$ will have the form of I-i, I-ii, I-iii or I-iv. If $J$ does not contain a linear form, then since $e\left(S /(x, y, z, w)^{2}\right)=5$, there exists three generators of the form $a x+b y+c z+d w, e x+$ $f y+g z+h w$ and $k x+l y+m z+n w$. We may assume that these generators are linearly independent or else $e(S / J)>2$. By lemma A.1, the ideal $(x, y, z, w)^{2}+(a x+b y+c z+d w, e x+f y+g z+h w, k x+l y+m z+n w)$ 
ON THE PROJECTIVE DIMENSION OF 5 QUADRIC ALMOST COMPLETE INTERSECTIONS WITH LOW MULTIPLICITIES7

is unmixed with multiplicity 2 , and hence equal to $J$ if ht $\left(x, y, z, w, I_{3}\left(\begin{array}{cccc}a & b & c & d \\ e & f & g & h \\ k & l & m & n\end{array}\right)\right) \geq 6$. This puts us in case II-i. We may suppose that $\left.\operatorname{ht}\left(I_{3}(M)\right)+(x, y, z, w)\right) /(x, y, z, w)=1$. By lemma 3.2, $M$ can take six different forms. When $M$ has the form of 3.2. , we have $a x \in J$ but $a \notin(x, y, z, w)$ and $J$ is $(x, y, z, w)$ primary then $x \in J$ and $J$ contains a linear form. When $M$ has the form of 3.2.ii, we have $(a f-b e) y \in J$ and $(a f-b e) \notin(x, y, z, w)$ hence $y \in J$, also a contradiction. Similarly, when $M$ has the form of 3.2 .v, we get $((a f-b e)(a m-k c)-(a l-k b)(a g-c e)) z=a(\operatorname{det}(N)) z \in J$, with $N$ obtained from $M$ by deleting the $4^{t h}$ column. However, $\operatorname{adet}(N) \notin(x, y, z, w)$ hence $z \in J$, which is a contradiction as well. Suppose $M$ has the form of 3.2.vi. By Remark 3.3-i, either $J=(x, y, z, w)^{2}+(a x+b y, k z+l w, l y+a z, k x+b w, b z-l x, a w-k y)$ by Lemma A.40 or $J$ contains a linear form. When $(x, y, z, w)^{2}+(a x+b y, k z+a w, k x+l y+l z+b w)$ in Remark 3.3-ii, we get $(a l-b k)(z+y) \in J$ and $z+y \in J$. Hence $J$ contains a linear form, which is impossible. We conclude that, when $M$ has the form of 3.2.vi, all quadrics are written in terms of 8 variables which puts us in case II-iv. The remaining cases for $M$ are 3.2.iii and 3.2.iv. In the latter case, we get $J=(x, y, z, w)^{2}+(a x+b y, e x+f y+n z, k x+l y+n w)$ and $a n((a l-b k) z-(a f-b e) w) \in J$. Since an $\notin(x, y, z, w)$, then $(a l-b k) z-(a f-b e) w \in J$. Consider the following ideal $K=(x, y, z, w)^{2}+(a x+$ $b y, e x+f y+n z, k x+l y+n w,(a l-b k) z-(a f-b e) w)$ with ht $\left(x, y, z, w, n, I_{2}\left(\begin{array}{ll}a & b \\ e & f \\ k & l\end{array}\right)\right)=7$, or else $K$ contains a linear form. By lemma A.2, $K$ is unmixed of multiplicity 2 and is equal to $J$, leaving us in case

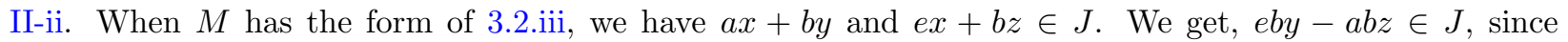
$b \notin(x, y, z, w)$ and $J$ is primary then $e y-a z \in J$. We have ht $(a, b, e)=3$, or else $J$ contains a linear form. By Lemma A.3, the ideal $(x, y, z, w)^{2}+(a x+b y, e x+b z, e y-a z, k x+l y+m z+n w)$ is unmixed of height 4 and multiplicity 2 , hence it is equal to $J$. This leaves us in II-iii.

\section{4. $(x, y, z, w)$-PRIMARY OF MULTIPLICITY 3}

In this section, we give a characterization of the degree 2 component of an ideal primary to the linear prime $\mathfrak{p}=(x, y, z, w)$ of multiplicity 3 . Before stating our result, we again prove a lemma on matrices with linear forms. We start with the following:

Lemma 4.1. Let $M=\left(\begin{array}{llll}a & b & c & d \\ e & f & g & h\end{array}\right)$ with $h t\left(I_{2}(M)\right)=2$ then $M$ is one of the following:

(i) $M=\left(\begin{array}{llll}a & b & 0 & 0 \\ e & f & g & h\end{array}\right)$ with $h t(a, b)=2, h t(g, h)>0$ and $h t(e, f, g, h) \geq 2$.

(ii) $M=\left(\begin{array}{llll}a & b & c & 0 \\ e & f & g & 0\end{array}\right)$ with $h t(a, b, c)=h t(e, f, g)=3$.

(iii) $M=\left(\begin{array}{llll}a & b & c & 0 \\ e & f & 0 & c\end{array}\right)$ with $h t(a f-b e, c)=2, h t(a, b, c)=h t(e, f, c)=3$.

(iv) $M=\left(\begin{array}{llll}a & b & c & 0 \\ e & 0 & b & c\end{array}\right)$ with $h t(a, b, c, e)=4$.

Proof. Since $\operatorname{ht}\left(I_{2}(M)\right)=2$, then $M$ is not 1-generic and has a generalized zero. So we may assume that $d=0$ and $M=\left(\begin{array}{llll}a & b & c & 0 \\ e & f & g & h\end{array}\right)$. We get $I_{2}(M)=(a f-b e, a g-e c, b g-f c, a h, b h, c h)=(a, b, c) \cap$ $\left(h, I_{2}\left(\begin{array}{lll}a & b & c \\ e & f & g\end{array}\right)\right)$. Hence the following cases may occur:

- $\operatorname{ht}(a, b, c)=2$, which puts us in i

- $h=0$, which is case ii

- $\operatorname{ht}(a, b, c)=3, \operatorname{ht}(e, f, g, h) \geq 3$ and $I_{2}(M)$ is contained in a prime of height two.

We study the last case in detail. The primes of height two that contain $I_{2}(M)$ are either obtained from two linear forms or from one linear form and one quadric. The linear forms are namely coming from $a, b, c, e, f, g, h$ 
and the quadric one from $I_{2}\left(\begin{array}{lll}a & b & c \\ e & f & g\end{array}\right)$. Since $\operatorname{ht}(a, b, c)=3$ and $\operatorname{ht}(e, f, g, h) \geq 3$, we only study the cases when the primes containing $I_{2}(M)$ are $(a, c)$ or $(h, a f-b e)$. All other cases lead us back to these two cases, either by a linear change of variables or by studying the generators of $I_{2}(M)$. For instance, if we are studying the case when $I_{2}(M) \subset(a, e)$ then $b g, f c, b h, c h \in(a, e)$. Since ht $(a, b, c)=3$ and ht $(e, f, g, h) \geq 3$, then either $b$ or $c$ are in $(e)$. We may assume $c=e$. We show below that if $I_{2}(M) \subset(a, c)$ then either $e=c$ or $e=0$. Hence, since ht $\left(I_{2}(M)\right)=2$, then the case when $I_{2}(M) \subset(a, e)$ is be equivalent to the case when $I_{2}(M) \subset(a, c)$.

Suppose $I_{2}(M) \subset(a, c)$. Since $\operatorname{ht}(a, b, c)=3$, we get $e, g, h \in(a, c)$. We assume $h \neq 0$ or else we are in ii. The cases when $h=a$ and $h=c$ are identical so we study one of them in detail. Suppose $h=a$, then we may assume that $g=0$ by row/column operations and $e=c$. Hence we get to iv by row/column operations. We note, that when $h=c$, we get $e=0$ and $g=a$. We again obtain iv by row/column operations. When $I_{2}(M) \subset(a f-b e, h)$, we have $b g-c f, a g-c e \in(a f-b e, h)$. This puts us either in i or in iii when $g=0$ and $c \in h$. We note that we consider $\operatorname{ht}(a, b, c)=\operatorname{ht}(e, f, c)=3$ in iii and $\operatorname{ht}(a, b, c, e)=4$ in iv, or else we go back to i.

Lemma 4.2. Let $M=\left(\begin{array}{cccc}a & b & c & d \\ e & f & g & h \\ k & l & m & n\end{array}\right)$ with $h t\left(I_{3}(M)\right)=h t\left(I_{2}(M)\right)=2$. Suppose ht $(a, b, c) \geq 2$ and $d=0$, then $M$ is one of the following:

(i) $M=\left(\begin{array}{cccc}a & b & 0 & 0 \\ 0 & a & b & 0 \\ k & l & m & n\end{array}\right)$ with $n \neq 0, \operatorname{ht}(a, b, k, l, m, n) \geq 5$.

(ii) $M=\left(\begin{array}{llll}a & b & 0 & 0 \\ 0 & 0 & a & b \\ k & l & m & n\end{array}\right)$ with $h t(a, b, k, l, m, n) \geq 5$.

(iii) $M=\left(\begin{array}{llll}a & b & c & 0 \\ e & f & g & 0 \\ k & 0 & f & g\end{array}\right)$ or $M=\left(\begin{array}{llll}a & b & c & 0 \\ e & f & g & 0 \\ k & 0 & g & f\end{array}\right)$ with $h t(a, e, f, g, k)=5$ and $b, c \in$ (f,g) (one of b or $c \neq 0)$.

(iv) The form of $M$ is determined by at most four variables.

Proof. Since $\operatorname{ht}\left(I_{3}(M)\right)=\operatorname{ht}\left(I_{2}(M)\right)=2$, we first show that $\operatorname{ht}\left(I_{2}\left(M_{i}\right)\right)=2$ for all $i$ where $M_{1}=$ $\left(\begin{array}{llll}a & b & c & d \\ e & f & g & h\end{array}\right), M_{2}=\left(\begin{array}{cccc}a & b & c & d \\ k & l & m & n\end{array}\right), M_{3}=\left(\begin{array}{cccc}e & f & g & h \\ k & l & m & n\end{array}\right)$. For that, if there exists an $i$ such that $\operatorname{ht}\left(I_{2}\left(M_{i}\right)\right)=3$, then $\operatorname{ht}\left(I_{2}(M)\right) \geq \operatorname{ht}\left(I_{2}\left(M_{i}\right)\right)=3$ which is impossible. If there exists an $i$ such that $\operatorname{ht}\left(I_{2}\left(M_{i}\right)\right)=1$, then $M_{i}$ is one of Lemma 3.1 which implies that $\operatorname{ht}\left(I_{3}(M)\right)=1$.

Since $\operatorname{ht}\left(I_{2}\left(M_{1}\right)\right)=2$, then $M_{1}$ is one of the cases of Lemma 4.1 and $M$ takes one of the following forms: 1) $\left.\left.M=\left(\begin{array}{cccc}a & b & 0 & 0 \\ e & f & g & h \\ k & l & m & n\end{array}\right), 2\right) M=\left(\begin{array}{cccc}a & b & c & 0 \\ e & f & g & 0 \\ k & l & m & n\end{array}\right), 3\right) M=\left(\begin{array}{cccc}a & b & c & 0 \\ e & f & 0 & c \\ k & l & m & n\end{array}\right)$ or 4$) M=\left(\begin{array}{cccc}a & b & c & 0 \\ e & 0 & b & c \\ k & l & m & n\end{array}\right)$

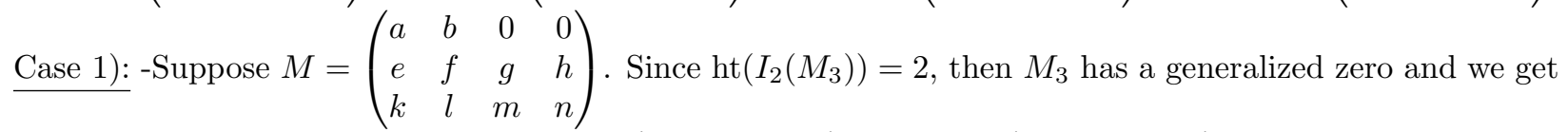
two forms for the matrix $M$ : either $M=\left(\begin{array}{cccc}a & b & 0 & 0 \\ e & f & g & 0 \\ k & l & m & n\end{array}\right)$ or $M=\left(\begin{array}{cccc}0 & 0 & a & b \\ e & f & g & 0 \\ k & l & m & n\end{array}\right)$. We divide the rest of the proof into two parts:

1a) $\operatorname{ht}(e, f, g)=2$.

1b) $\operatorname{ht}(e, f, g)=3$ and $\operatorname{ht}(k, l, m, n) \geq 3$. 
ON THE PROJECTIVE DIMENSION OF 5 QUADRIC ALMOST COMPLETE INTERSECTIONS WITH LOW MULTIPLICITIES9

1a) ht $(e, f, g)=2$ : We suppose $M=\left(\begin{array}{llll}a & b & 0 & 0 \\ e & f & g & 0 \\ k & l & m & n\end{array}\right)$ with $n \neq 0$ and $g \neq 0$ or else $\operatorname{ht}\left(I_{3}(M)\right)=1$. We can also assume that either $e=0$ or $f=0$ by a column operation and a linear change of variables. We write $M=\left(\begin{array}{cccc}a & b & 0 & 0 \\ 0 & f & g & 0 \\ k & l & m & n\end{array}\right)$

- If $\operatorname{ht}(a, b, f, g)=2$, then $M=\left(\begin{array}{cccc}a & b & 0 & 0 \\ 0 & a & b & 0 \\ k & l & m & n\end{array}\right)$ with $n \neq 0$. We may assume that $\operatorname{ht}(a, b, k, l, m, n) \geq 5$ or else we are back to iv.

- If ht $(a, b, f, g)=3$, then the cases $f \in(a)$ or $g \in(b)$ are identical after a linear change of variable, which reduces our study to the following three case: $f \in(a), f \in(b)$ or $g \in(a)$. When $g \in(a)$, we get ht $\left(I_{3}(M)\right)=$ 1 , which is impossible. If $f \in(a)$, then $M=\left(\begin{array}{cccc}a & b & 0 & 0 \\ 0 & a & g & 0 \\ k & l & m & n\end{array}\right)$ with $I_{3}(M)=\left(a^{2} n, a g n, b g n, a^{2} m-a g l+b g k\right)=$ $(a, b) \cap\left(a^{2}, g\right) \cap\left(n, a^{2} m-a g l+b g k\right)$ and $I_{2}(M)=\left(a^{2}, a l-b k, a k, a g, b g, g k, g l, a m, b m, a n, b n, g n\right)=(a, b, g) \cap$ $\left(a^{2}, k^{2}, a k, g, m, n, a l-b k\right) \cap(a, b, k, l, n)$. Since ht $(a, b, g)=3$ and $a^{2}, b g \in I_{2}(M)$, then the only two cases to study are whenever $I_{2}(M) \subset(a, b)$ or $(a, g)$. The other cases give us either ht $(a, b, g)<3$ or ht $\left(I_{3}(M)\right)=1$. If $I_{2}(M) \subset(a, b)$, then $g k, g l, g n \in(a, b)$ which implies $k, l, n \in(a, b)$. Similarly when $I_{2}(M) \subset(a, g)$, we get $k, m, n \in(a, g)$. Both cases put us in iv. Now, we suppose that $f \in(b)$, then $M=\left(\begin{array}{cccc}a & b & 0 & 0 \\ 0 & b & g & 0 \\ k & l & m & n\end{array}\right)$. We repeat the same study as above. We have $I_{3}(M)=(b g k-a g l+a b m, a b n, a g n, b g n)=(a, b) \cap(a, g) \cap$ $(b, g) \cap(n, a b m-a g l+b g k)$ and $I_{2}(M)=(a b, a l-b k, b k, a g, a m, g k, b g, b m, b m-g l, a n, b n, b n, g n)=(a, b, g) \cap$ $(a, g, k, m, n) \cap(b, g, l, m, n) \cap(a, b, k, l, n)$. Again, since $\mathrm{ht}(a, b, g)=3$ and $a g, b g \in I_{2}(M)$ then we study the cases when $I_{2}(M) \subset(a, b),(a, g)$ or $(b, g)$. We get $k, l, n \in(a, b), k, m, n \in(a, g)$ or $l, m, n \in(b, g)$ respectively. This puts us one more time in iv.

- If $\operatorname{ht}(a, b, f, g)=4$, then $M=\left(\begin{array}{cccc}a & b & 0 & 0 \\ 0 & f & g & 0 \\ k & l & m & n\end{array}\right)$ with $I_{2}(M)=(a f, a l-b k, f k, a g, a m, g k, b g, b m, f m-$ $g l, a n, b n, f n, g n)=(a, b, f, g) \cap(a, b, k, n, f m-g l) \cap(a, g, k, m, n) \cap(a l-b k, f, g, m, n)$ Since ht $(a, b, f, g)=4$ and $a f, a g, b g \in I_{2}(M)$ then the only cases to study are when $I_{2}(M) \subset(a, b),(a, g)$ or $(b, g)$, we note that it would be impossible for $I_{2}(M)$ to be a subset of $(a, f),(b, g)$ nor $(b, f)$. If $I_{2}(M) \subset(a, b)$, then $f k, f m, f n, g l, g k, g n \in(a, b)$ which implies $k, l, m, n \in(a, b)$. This puts us in iv. If $I_{2}(M) \subset(a, g)$, then $f k, f m, f n, b k, b m, b n, \in(a, g)$ and $k, m, n \in(a, g)$. Hence the only two possibilities for $M$ are $M=$ $\left(\begin{array}{llll}a & b & 0 & 0 \\ 0 & f & g & 0 \\ 0 & l & a & g\end{array}\right)$ or $M=\left(\begin{array}{llll}a & b & 0 & 0 \\ 0 & f & g & 0 \\ g & l & 0 & a\end{array}\right)$, otherwise $h t\left(I_{3}(M)\right)=1$. We can rewrite both matrices $M$, after row/column operations and a linear change of variables, as $M=\left(\begin{array}{cccc}b & a & 0 & 0 \\ f & a & g & 0 \\ l & 0 & a & g\end{array}\right)$ or $M=\left(\begin{array}{cccc}b & 0 & a & 0 \\ f & g & a & 0 \\ l & 0 & g & a\end{array}\right)$ respectively. Both matrices are reduced to iii. Finally, the case when $I_{2}(M) \subset(f, g)$ is identical to $I_{2}(M) \subset(a, b)$ by a linear change of variables.

-We then study the case when $M=\left(\begin{array}{cccc}0 & 0 & a & b \\ e & f & g & 0 \\ k & l & m & n\end{array}\right)$. Since ht $(e, f, g)=2$, we assume $g=0$. We write $M=\left(\begin{array}{cccc}a & b & 0 & 0 \\ 0 & 0 & e & f \\ k & l & m & n\end{array}\right)$ 
- If $\operatorname{ht}(a, b, e, f)=2$, then $M=\left(\begin{array}{cccc}a & b & 0 & 0 \\ 0 & 0 & a & b \\ k & l & m & n\end{array}\right)$ with $h t(a, b, k, l, m, n) \geq 5$ which is case ii.

- If $\operatorname{ht}(a, b, e, f)=3$, then we may assume $e \in(a)$, and $M=\left(\begin{array}{cccc}a & b & 0 & 0 \\ 0 & 0 & a & f \\ k & l & m & n\end{array}\right)$. Since $a b, a f$ and $a^{2} \in$ $I_{2}(M)=\left(a l-b k, a^{2}, a m, a k, a b, b m, a l, a f, a n, f k, b f, b n, f l, a n-f m\right)=(a, b, f) \cap(a, b, k, l, m) \cap(a, f, k, m, n) \cap$ $\left(a^{2}, b, k, f, l, m, n\right)$ and $\mathrm{ht}(a, b, f)=3$ then the only cases to study are whenever $I_{2}(M) \subset(a, b)$ or $(a, f)$. If $I_{2}(M) \subset(a, b)$ or $(a, f)$, then $f m, f k, f l \in(a, b)$ or $b k, b m, b n \in(a, f)$ respectively. Hence $k, l, m \in(a, b)$ or $k, m, n \in(a, f)$, and we get back to iv.

- If $\operatorname{ht}(a, b, e, f)=4$, then we write $M=\left(\begin{array}{cccc}a & b & 0 & 0 \\ 0 & 0 & e & f \\ k & l & m & n\end{array}\right)$. Similarly to the above cases, we have $a e, a f, b e, b f \in I_{2}(M)=(a l-b k, a e, a m, e k, b e, b m, e l, a f, a n, f k, b f, b n, f l, e n-f m)=(a, b, e, f) \cap(a l-$ $b k, e, f, m, n) \cap(f m-e n, a, b, k, l)$. We only study the case when $I_{2}(M) \subset(a, b)$. We get $k, l, m, n \in(a, b)$. This puts us in case iv.

1b) $\underline{\text { ht }(e, f, g)=3 \text { and ht }(k, l, m, n) \geq 3:}$-Suppose $M=\left(\begin{array}{cccc}a & b & 0 & 0 \\ e & f & g & 0 \\ k & l & m & n\end{array}\right)$ with $n \neq 0$ and $I_{2}(M)=(a f-$ $b e, a l-b k, e l-f k, e m-g k, f m-g l, a g, a m, b g, b m, a n, b n, e n, f n, g n)$. We begin by simplifying the matrix $M$ by looking at $M_{3}$. We know that $\operatorname{ht}\left(I_{2}\left(M_{3}\right)\right)=2$, so $I_{2}\left(M_{3}\right)$ should be included in a height two prime ideal which is either generated by two linear forms or by one linear and one quadric. Looking at the form of the matrix $M$, we study the cases when $I_{2}\left(M_{3}\right) \subset(e, f),(e, g),(e l-f k, n)$ and $(e m-g k, n)$. All other cases put us in these mentioned cases or back to 1a). Suppose $I_{2}(M) \subset(e, f)$, then $n \in(e, f)$ and $g k, g l \in(e, f)$. We only study the case when $n=e$, since when $n=f$ is similar. Suppose $n=e$, then $k, l \in(e, f)$. We may assume that $l=0$ by a row/column operations, and the only possibility for $M$ is $M=\left(\begin{array}{cccc}a & b & 0 & 0 \\ e & f & g & 0 \\ f & 0 & m & e\end{array}\right)$. We write $M=\left(\begin{array}{cccc}0 & 0 & a & b \\ m & e & f & 0 \\ g & 0 & e & f\end{array}\right)$ after row/column operations, and we look at $I_{2}(M)$. We have $a g, b g \in I_{2}(M)$, which implies that $a, b \in(e, f)$. Hence we either get back to 1a) or we get ht $\left(I_{2}(M)\right)=1$ after row/column operations. Suppose $I_{2}\left(M_{3}\right) \subset(e, g)$, then $f k, f m, f n \in(e, g)$. Suppose $n=e$. We may assume that $m=0$ and $k=g$. Hence $M=\left(\begin{array}{cccc}a & b & 0 & 0 \\ e & f & g & 0 \\ g & l & 0 & e\end{array}\right)$, which can be written as $M=\left(\begin{array}{cccc}b & 0 & a & 0 \\ l & e & g & 0 \\ f & 0 & e & g\end{array}\right)$ after row/column operations. Since $a f, a l \in I_{2}(M)$ then $a \in(e, g)$ which put us in iii. When $n=g$, we get $m=e$ and $k=0$. Hence $M=\left(\begin{array}{llll}b & a & 0 & 0 \\ f & e & g & 0 \\ l & 0 & e & g\end{array}\right)$ after row/column operations, and again $a \in(e, g)$. This puts us in iii. When $I_{2}\left(M_{3}\right) \subset(e l-f k, n)$, then either $M=\left(\begin{array}{llll}a & b & 0 & 0 \\ e & f & n & 0 \\ k & l & 0 & n\end{array}\right)$ or $M=\left(\begin{array}{llll}a & b & 0 & 0 \\ e & n & f & 0 \\ k & 0 & l & n\end{array}\right)$. In the former case, $\operatorname{ht}\left(I_{2}(M)\right)=1$ and we are done. In the latter case we get $n^{2} \in I_{2}(M)$. So we study the cases when $I_{2}(M) \subset(a, n),(b, n)$ or $(e l-f k, n)$. When $I_{2}(M) \subset(a, n)$ then $e l-f k \in(a, n)$. We may assume $f=k=a$ but $b l \in(a, n)$. Hence either $b=n$ or $l \in(a, n)$. We get back to case 1a. Similarly when $I_{2}(M) \subset(b, n)$, we get back to case 1a. When $I_{2}(M) \subset(e l-f k, n)$, we get $a f, b f \in I_{2}(M)$. Hence $a, b \in(n)$ and ht $\left(I_{2}(M)\right)=1$. The case when $I_{2}(M) \subset(e m-g k, n)$ is treated the same way as whenever $I_{2}(M) \subset(e l-f k, n)$, so we omit the details. 
ON THE PROJECTIVE DIMENSION OF 5 QUADRIC ALMOST COMPLETE INTERSECTIONS WITH LOW MULTIPLICITIE\$1

- Now we suppose $M=\left(\begin{array}{cccc}0 & 0 & a & b \\ e & f & g & 0 \\ k & l & m & n\end{array}\right)$. We conduct the same proof as above. $\operatorname{Since} \operatorname{ht}\left(I_{2}\left(M_{3}\right)\right)=2$ and ht $(e, f, g)=3$, we study the cases when $I_{2}\left(M_{3}\right) \subset(e, f),(e, g),(e m-g k, n)$ and $(e l-f k, n)$. When $I_{2}\left(M_{3}\right) \subset(e, f)$, we get $g l, g k, g n \in(e, f)$ and hence $k, l, n \in(e, f)$. We may assume that $n \neq 0$ since otherwise we go back to case 1a) after row/column operations because $k, l \in(e, f)$. We assume $n=e$, the case when $n=f$ is similar and we will omit the details. When $n=e$, the two possibilities for the matrix $M$ would be $M=\left(\begin{array}{cccc}0 & 0 & a & b \\ e & f & g & 0 \\ f & 0 & m & e\end{array}\right)$ or $M=\left(\begin{array}{cccc}0 & 0 & a & b \\ e & f & g & 0 \\ 0 & f & m & e\end{array}\right)$. These matrices can be written as $M=\left(\begin{array}{cccc}a & b & 0 & 0 \\ m & e & f & 0 \\ g & 0 & e & f\end{array}\right)$ and $M=\left(\begin{array}{cccc}a & b & 0 & 0 \\ m & e & f & 0 \\ g & 0 & f & e\end{array}\right)$ after row/column operation. Since $b g, b m \in(e, f)$ then $b \in(e, f)$, and this puts us in iii. When $I_{2}\left(M_{3}\right) \subset(e, g)$, then $k, m, n \in(e, g)$. Again $n \neq 0$ and we

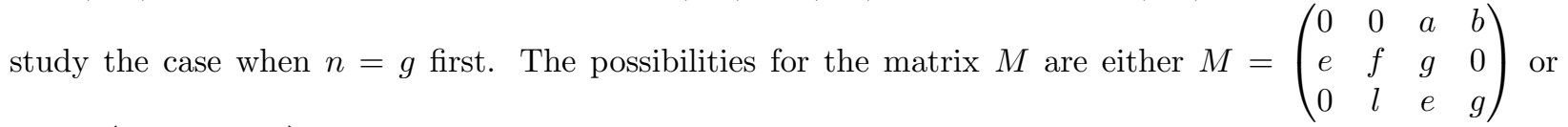
$M=\left(\begin{array}{cccc}b & 0 & a & b \\ e & f & g & 0 \\ 0 & l & e & g\end{array}\right)$. In the former case we get $(a, b) \subset(e, g)$ and hence we are either back to case 1a) after row/column operations or we get $h t\left(I_{2}(M)\right)=1$. In the latter case, we also get $(a, b) \subset(e, g)$ and back to case 1a) after row/column operations. When $n=e$, we get $M=\left(\begin{array}{llll}0 & 0 & a & b \\ e & f & g & 0 \\ g & l & 0 & e\end{array}\right)$ and $(a, b) \subset(e, g)$. We get back to case 1a) after row/column operations.

When $I_{2}\left(M_{3}\right) \subset(e l-f k, n)$, then either $M=\left(\begin{array}{cccc}0 & 0 & a & b \\ e & n & f & 0 \\ k & 0 & l & n\end{array}\right)$ or $M=\left(\begin{array}{llll}0 & 0 & a & b \\ e & f & n & 0 \\ k & l & 0 & n\end{array}\right)$. In both cases, we have $n^{2} \in I_{2}(M)$. So we study the cases when $I_{2}(M) \subset(a, n),(b, n)$ and $(e l-f k, n)$. In both matrices, when $I_{2}(M) \in(a, n)$ or $(b, n)$, we get to case back 1a. When $I_{2}(M) \subset(e l-f k, n)$, we get $a, b \in(n)$ and we get $\operatorname{ht}\left(I_{2}(M)\right)=1$. The case when $I_{2}\left(M_{3}\right) \subset(e m-g k, n)$ is identical and treated the same way as $I_{2}\left(M_{3}\right) \subset(e l-f k, n)$, so we omit the details.

$\underline{\text { Case 2): }}$ Suppose $M=\left(\begin{array}{cccc}a & b & c & 0 \\ e & f & g & 0 \\ k & l & m & n\end{array}\right)$. Again we have $\operatorname{ht}\left(I_{2}\left(M_{1}\right)\right)=\operatorname{ht}\left(I_{2}\left(M_{3}\right)\right)=2$. We may assume that $\operatorname{ht}(a, b, c)=\operatorname{ht}(e, f, g)=3$ and $\operatorname{ht}(k, l, m, n) \geq 3$, or else we are back to Case 1 . Since ht $\left(I_{2}\left(M_{3}\right)\right)=2$, then we study the cases when $I_{2}\left(M_{3}\right) \subset(e, f)$ and $(e l-f k, n)$. We follow the same argument as before. If $I_{2}\left(M_{3}\right) \subset(e, f)$, then $k, l, n \in(e, f)$ and we study the case when $n=e$ only (the case $n=f$ is identical). Hence, $M=\left(\begin{array}{cccc}a & b & c & 0 \\ e & f & g & 0 \\ f & 0 & m & e\end{array}\right)$ written as $M=\left(\begin{array}{cccc}c & b & a & 0 \\ g & f & e & 0 \\ m & 0 & f & e\end{array}\right)$. We get $a g, a m, b g, b m \in(e, f)$ and hence $a, b \in(e, f)$. This puts us in iii. When $I_{2}\left(M_{3}\right) \subset(e l-f k, n)$, we write $M=\left(\begin{array}{cccc}a & b & c & 0 \\ e & f & n & 0 \\ k & l & 0 & n\end{array}\right)$. We have $n^{2} \in I_{2}(M)$. So we investigate the cases whenever $I_{2}(M) \subset(a, n),(c, n),(a f-b e, n)$ and $(e l-f k, n)$. When $I_{2}(M) \in(a, n),(a, c)$ or $(a f-b e, n)$, we get that $e l-f k$ belongs each of these prime ideals respectively. All these cases put us back in Case 1 . When $I_{2}(M) \in(e l-f k, n)$, we get $c \in(n)$ since $c k, c l \in(n)$ and $\operatorname{ht}\left(I_{2}(M)\right)=1$. 
Case 3): Suppose $M=\left(\begin{array}{cccc}a & b & c & 0 \\ e & f & 0 & c \\ k & l & m & n\end{array}\right)$. We may also assume ht $(a, b, c)=\operatorname{ht}(e, f, c)=3$ and ht $(k, l, m, n) \geq$ 3 , or else we get back to Case 1. We have ht $\left(I_{2}\left(M_{3}\right)\right)=2$, and again we study the cases when $I_{2}\left(M_{3}\right) \subset$ $(e, f),(e, c)$ and $(e l-f k, c)$. Suppose $I_{2}\left(M_{3}\right) \subset(e, f)$, then since $c^{2} \in I_{2}(M)$ we get $c \in(e, f)$ which puts is back in Case 1. If $I_{2}\left(M_{3}\right) \subset(e, c)$, then $f k, f m, f n \in(e, c)$ and $k, m, n \in(e, c)$. Suppose $m=c$ then $m=0$ after row operation and a linear change of variables. If $n=c$ then $\operatorname{ht}\left(I_{3}(M)\right)=1$, so we assume $n=e$. We also assume that $k=c$ or else we get back to Case 1. Hence $M=\left(\begin{array}{cccc}a & b & c & 0 \\ e & f & 0 & c \\ c & l & 0 & e\end{array}\right)$ and again we go back to Case 1 after row/column operation. We now suppose $m=e$, then $n=0$ by row/column operations and $k=c$ or else we get back to Case 1. Hence $M=\left(\begin{array}{cccc}a & b & c & 0 \\ e & f & 0 & c \\ c & l & e & 0\end{array}\right)$. Since al, af $\in(e, c)$ and ht $(e, f, c)=\operatorname{ht}(c, e, l)=3$, then $a \in(e, c)$ which puts us in any of the two forms of iii. Finally suppose $I_{2}\left(M_{3}\right) \subset(e l-f k, m)$, then since $c^{2} \in I_{2}(M)$ we get $c=m$. We only study the case when $I_{2}(M) \subset(e l-f k, m)$ since otherwise we get back to Case 1. We have $b n \in I_{2}(M)$ and ht $(a, b, c)=3$, so $n=m$ and hence $n=0$. So ht $\left(I_{3}(M)\right)=1$ whenever $M=\left(\begin{array}{cccc}a & b & m & 0 \\ e & f & 0 & m \\ k & l & m & 0\end{array}\right)$.

Case 4:) Suppose $M=\left(\begin{array}{cccc}a & b & c & 0 \\ e & 0 & b & c \\ k & l & m & n\end{array}\right)$. In this case, we do not need to study the cases when $I_{2}\left(M_{3}\right) \subset$ $(e, b),(e, c),(b, c),(b n-m c, l)$ since $b^{2}, c^{2} \subset I_{2}(M)=$. Hence we study the case when $I_{2}(M) \subset(b, c)$. We get $l, m, n \in(b, c)$ and we are in iii after row/column operations.

Remark 4.3. We record the matrices of 4.2-iv as details are needed to complete the proof of Proposition 4.4:

(i) $M=\left(\begin{array}{cccc}a & b & 0 & 0 \\ 0 & a & b & 0 \\ k & l & m & n\end{array}\right)$ or $M=\left(\begin{array}{llll}a & b & 0 & 0 \\ 0 & 0 & a & b \\ k & l & m & n\end{array}\right)$ with $\left\{\begin{array}{c}\operatorname{ht}(a, b, n)=3, \text { and } 3 \leq \mathrm{ht}(a, b, k, l, m, n) \leq 4 \text { or } \\ n=a, \operatorname{ht}(k, n)=2, \text { and } 3 \leq \mathrm{ht}(a, b, k, l, m, n) \leq 4 \text { or } \\ n=b, \operatorname{ht}(m, n)=2, \text { and } 3 \leq \operatorname{ht}(a, b, k, l, m, n) \leq 4 .\end{array}\right.$

(ii) $M=\left(\begin{array}{llll}a & b & 0 & 0 \\ 0 & a & b & 0 \\ 0 & 0 & a & b\end{array}\right)$ with $\operatorname{ht}(a, b)=2$.

(iii) $M=\left(\begin{array}{cccc}d & m & 0 & 0 \\ c & b & a & 0 \\ 0 & 0 & b & a\end{array}\right)$ or $M=\left(\begin{array}{cccc}d & m & 0 & 0 \\ c & 0 & b & a \\ 0 & b & a & 0\end{array}\right)$ with $m \in(a, b)$ and $\operatorname{ht}(a, b, c, d)=4$

(iv) $M=\left(\begin{array}{llll}a & b & 0 & 0 \\ c & b & a & 0 \\ d & 0 & b & a\end{array}\right)$ or $M=\left(\begin{array}{cccc}a & 0 & b & 0 \\ c & b & a & 0 \\ d & 0 & b & a\end{array}\right)$ with $\operatorname{ht}(a, b, c)=\operatorname{ht}(a, b, d)=3$

(v) $M=\left(\begin{array}{llll}d & a & m & 0 \\ c & b & a & 0 \\ 0 & 0 & a & b\end{array}\right)$ or $M=\left(\begin{array}{cccc}d & b & m & 0 \\ c & 0 & b & a \\ 0 & a & b & 0\end{array}\right)$ with $m \in(a, b)$ and $\operatorname{ht}(a, b, c, d)=4$.

Proposition 4.4. Let $J$ be an $(x, y, z, w)$-primary with $e(S / J)=3$. Then one of the following holds

I- If $J$ is degenerate, then

(i) $J=\left(x, y, z, w^{3}\right)$.

(ii) $J=(x, y)+(z, w)^{2}$.

(iii) $J=(x, y)+\left(z^{2}, w z, w^{3}, w^{2}+a z\right)$ with $h t(x, y, z, w, a)=5$.

(iv) $J=(x, y)+\left(z^{3}, z^{2} w, z w^{2}, w^{3}, a z+b w\right)$ with $h t(x, y, z, a, b)=5$.

(v) $J=(x)+(y, z, w)^{2}+(b y+c z+d w)$ with $h t(x, y, z, w, b, c, d) \geq 6$. 
ON THE PROJECTIVE DIMENSION OF 5 QUADRIC ALMOST COMPLETE INTERSECTIONS WITH LOW MULTIPLICITIE\$3

(vi) $J=(x)+(y, z)^{2}+\left(w y, w z, w^{2}+b y+c z, e y+f z\right)$ with $h t(x, y, z, w, e, f)=6$

(vii) $J=(x)+y(y, z, w)+(z, w)^{3}+\left(a y+b z+c w, d y+z^{2}\right)$ with $h t(x, y, z, w, b, c)=$ $h t(x, y, z, w, c, d)=6$.

(viii) $J=(x)+y(y, z, w)+(z, w)^{3}+\left(a y+b z+c w, c y+z^{2}, b y-z w\right)$ with $h t(x, y, z, w, b, c)=6$.

(ix) $J=(x)+y(y, z, w)+(z, w)^{3}+(a y+b z+c w, d y+z w)$ with $h t(x, y, z, w, d, c, d)=7$.

(x) $J=(x)+y(y, z, w)+(z, w)^{3}+\left(a y+b z+c w, b y+z w, c y-z^{2}\right)$ with ht $(x, y, z, w, b, c)=6$.

(xi) $J=(x)+y(y, z, w)+(z, w)^{3}+\left(a y+b z+c w, c y+z w, b y-w^{2}\right)$ with $h t(x, y, z, w, b, c)=6$.

(xii) All quadrics in $J$ generate an ideal of height at most three.

(xiii) All quadrics in $J$ can be expressed in terms of at most 8 variables.

II- If $J$ is non-degenerate, then

(i) $J=(x, y, z, w)^{2}+(a x+b y+c z+d w, e x+f y+g z+h w)$ with $h t\left(I_{2}\left(\begin{array}{llll}a & b & c & d \\ e & f & g & h\end{array}\right)\right) \geq 2$.

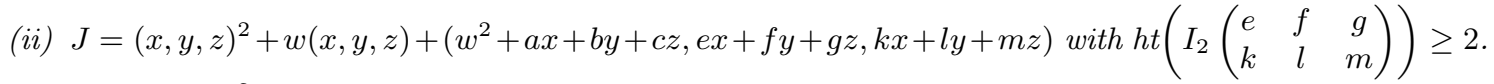

(iii) $J=(x, y, z)^{2}+w(x, y, z, w)+(a x+b y+c z, e x+f y, k x+f z, k y-e z)$ with $h t(e, f, k)=3$.

(iv) $J=(x, y)^{2}+(x, y)(z, w)+\left(a x+b y+c z+d w, e x+f y+z^{2}+\alpha w^{2}, g x+h y+z w\right)$ with $h t(x, y, z, w, c, d)=6$, $h t(c, g, h) \geq 2, h t(d, g, h) \geq 2, h t(e, f, g, h) \geq 2$ and $h t\left(I_{2}\left(\begin{array}{ll}e & f \\ g & h\end{array}\right)\right)=1,(\alpha \in k)$.

(v) $J=(x, y)^{2}+(x, y)(z, w)+\left(a x+b y+c z+d w, e x+z^{2}+w^{2}, e y+z w, c^{2} y+c d x+d^{2} y\right)$ with $h t(c, d, e)=3$.

(vi) $J=(x, y)^{2}+(x, y)(z, w)+\left(a x+b y+c z+d w, e x+z^{2}, e y+z w, c x+d y\right)$ with $h t(c, d, e)=3$.

(vii) $J=(x, y)^{2}+(x, y)(z, w)+\left(a x+b y+c z+d w, e x+f y+z^{2}+\alpha w^{2}, c x+z w, d x-z^{2}\right)$ with $h t(c, d)=h t(e, f)=2$.

(viii) $J=(x, y)^{2}+(x, y)(z, w)+\left(a x+b y+c z+d w, e x+f y, g x+h y+z^{2}\right)$ with $h t(c, d)=h t(e, f)=2$.

(ix) $J=(x, y)^{2}+(x, y)(z, w)+\left(a x+b y+c z+d w, e x+f y+w^{2}, g x+h y+z^{2}\right)$ with $h t(x, y, z, w, c, d)=6$, $h t(c, e, f) \geq 2, h t(d, g, h) \geq 2$ and $h t\left(I_{2}\left(\begin{array}{ll}e & f \\ g & h\end{array}\right)\right)=1$.

(x) $J=(x, y)^{2}+(x, y)(z, w)+\left(a x+b y+c z+d w, c x+w^{2}, g x+h y+z^{2}, d x-z w\right)$ with $h t(x, y, z, w, c, d)=6$ and $h t(d, g, h) \geq 2$.

(xi) All quadrics in $J$ generate an ideal of height at most three.

(xii) All quadrics in $J$ can be expressed in terms of at most 8 variables.

Proof. Since $J \subset J: \mathfrak{p}$ then $e(S / J)>e(S /(J: \mathfrak{p}))$. For that $e(S /(J: \mathfrak{p}))=1$ or 2 .

If $e(S /(J: \mathfrak{p}))=1$, then $(J: \mathfrak{p})=(x, y, z, w)$ and $(x, y, z, w)^{2} \subset J$. If $J$ contains three linear forms then $\left(x, y, z, w^{2}\right) \subset J$ and $e\left(S /\left(x, y, z, w^{2}\right)\right)=2 \geq e(S / J)$, which is a contradiction. If $J$ contains two linear forms then $J=(x, y)+(z, w)^{2}$, which puts us in case I-ii. If $J$ contains one linear form then $(x)+(y, z, w)^{2} \subset J$, and $J$ has at least one more element of the form $q=b y+c z+d w$. The ideal $(y, z, w)^{2}+(x, b y+c z+d w)$ is unmixed whenever $\operatorname{ht}(x, y, z, w, b, c, d) \geq 6$ by Lemma A.4, which is case I-v. If $h t(x, y, z, w, b, c, d)=5$, then we can modify $c, d$ modulo $(x, y, z, w)$ to assume that $c$ and $d$ are multiple of $b$. Hence $q=b l$ for some linear form $l$, and $J$ contains another linear form, which is a contradiction. If $J$ does not contain any linear form, then since $e\left(S /(x, y, z, w)^{2}\right)=5, J$ must contain at least two generators of the form $(a x+b y+c z+d w, e x+f y+g z+h w)$. If $h t\left(I_{2}\left(\begin{array}{llll}a & b & c & d \\ e & f & g & h\end{array}\right)\right) \geq 2$, then $(x, y, z, w)^{2}+(a x+b y+c z+d w, e x+f y+g z+h w)$ is unmixed of multiplicity 3 and hence equal to $J$ by Lemma A.5, which puts us in case II-i. When ht $\left(I_{2}\left(\begin{array}{llll}a & b & c & d \\ e & f & g & h\end{array}\right)\right)=1$ modulo $(x, y, z, w)$, then $M$ has one of the form of Lemma 3.1. In the first two cases, $J$ contains a linear form. In the last case, $(x, y, z, w)^{2}+(a x+b y, e x+b z) \subset J$ and $b(e y-a z) \in J$. Since $b \notin J$ then $e y-a z \in J$. Hence by lemma A. $6, J=(x, y, z, w)^{2}+(a x+b y, e x+b z, e y-a z)$, which puts us in II-xii.

Suppose $e(S /(J: \mathfrak{p})=2$ then there are 6 cases to consider according to Proposition 3.4. The case when all quadrics $J: \mathfrak{p}$ are written in terms of 8 variables put us in I-xiii or II-xii since $J \subset J: \mathfrak{p}$.

Case I. $J: \mathfrak{p}=\left(x, y, z, w^{2}\right)$

In this case, we have $(x, y, z)^{2}+\left(w x, w y, w z, w^{3}\right) \subset J \subset\left(x, y, z, w^{2}\right)$. If $J$ contains three independent linear 
forms then $J=\left(x, y, z, w^{3}\right)$, which is case I-i. If $J$ contains two linear forms say $x, y$ then $\left(x, y, z^{2}, w z, w^{3}\right) \subset$ $J$. Since this ideal on the left has multiplicity 4, then we need one additional generator of the form $w^{2}+a z$. By Lemma A.7, the ideal $\left(x, y, z^{2}, w z, w^{3}, w^{2}+a z\right)$ is unmixed of multiplicity 3 , and hence equal to $J$. If ht $(x, y, z, w, a)=5$ we are in case I-iii, otherwise when $a \in(x, y, z, w)$ we are in case I-ii. If $J$ contains a linear form $x$, then $(x)+(y, z)^{2}+\left(w y, w z, w^{3}\right) \subset J$. Since the multiplity of the ideal on the left is 5 , then either $J$ has a generator of the form $w^{2}+b y+c z+d w$ with $d \neq 0$, or two generators of the form $w^{2}+b y+c z$ and $e y+f z$. In the first case, we have $d w^{2} \in J$ since $d \notin(x, y, z, w)$ then $w^{2} \in J$. We get the ideal $(x, b y+c z+d w)+(w, y, z)^{2}$, which by lemma A.8, is unmixed of height four and multiplicity 3 when $\operatorname{ht}(x, y, z, w, b, c, d) \geq 6$. This puts us in case I-v, or back to I-ii when $\operatorname{ht}(x, y, z, w, b, c, d)=5$. In the second case, one of the quadrics must contain $w^{2}$, or else $J$ contains a second linear form. The ideal $(x)+(y, z)^{2}+\left(w y, w z, w^{2}+b y+c z, e y+f z\right)$ with $h t(x, y, z, w, e, f)=6$ is umixed of multiplicity 3 by Lemma A.9, and hence is equal to $J$. This puts us in case I-vi. If $\operatorname{ht}(x, y, z, w, e, f)=5$, then $J$ contains a second linear form $y$ or $z$. Suppose $J$ doesn't contain a linear form. Since the multiplicity of the ideal $(x, y, z)^{2}+\left(w x, w y, w z, w^{3}\right)$ is 6 , then $J$ must contain either two generators of the form $w^{2}+a x+b y+c z+d w$ and $e x+f y+g z+h w$ with $d \neq 0$ or $h \neq 0$, or three linearly independent quadrics of the from $w^{2}+a x+b y+c z$, $e x+f y+g z$ and $k x+l y+m z$. In the first case $w^{2} \in J$, and by lemma A.5, we are in case II-i when ht $\left(I_{2}\left(\begin{array}{cccc}a & b & c & d \\ e & f & g & h\end{array}\right)\right) \geq 2$. Otherwise, either $J$ contains a linear form or $J$ is in II-xii. In the second case, by Lemma A.10, the ideal $(x, y, z)^{2}+\left(w x, w y, w z, w^{2}, a x+b y+c z, e x+f y+g z, k x+l y+m z\right)$ is unmixed of multiplicity 3 when $h t\left(I_{2}\left(\begin{array}{ccc}e & f & g \\ k & l & m\end{array}\right)\right) \geq 2$ modulo $(x, y, z, w)$, and hence is equal to $J$. This puts us in case II-ii. If ht $\left(I_{2}\left(\begin{array}{ccc}e & f & g \\ k & l & m\end{array}\right)\right)=1$, then by [11, Lemma 4.1], $M=\left(\begin{array}{ccc}e & f & 0 \\ k & 0 & f\end{array}\right)$ with ht $(e, f, k)=3$ or else $J$ contains a linear form. In that case, $(x, y, z)^{2}+\left(w x, w y, w z, w^{2}+a x+b y+c z, e x+f y, k x+f z\right) \in J$ and $f(k y-e z) \in J$. Since $f \notin(x, y, z, w)$, then $k y-e z \in J$. By Lemma A.11, $(x, y, z)^{2}+\left(w x, w y, w z, w^{2}+a x+\right.$ $b y+c z, e x+f y, k x+f z, k y-e z)$ is unmixed of multiplicity 3 , and hence equal to J. This puts us in case II-iii.

Case II. $J: \mathfrak{p}=\left(x, y, z^{2}, z w, w^{2}, a z+b w\right), \operatorname{ht}(x, y, z, w, a, b)=6$.

In this case, $\left(x^{2}, x y, x z, x w, y^{2}, y z, y w, z^{3}, z^{2} w, z w^{2}, w^{3}, z(a z+b w), w(a z+b w)\right) \subset J \subset J: \mathfrak{p}$. If $J$ contains the two linear forms $x$ and $y$ then $\left(x, y, z^{3}, z^{2} w, z w^{2}, w^{3}, z(a z+b w), w(z a+b w)\right) \subset J$. There is at least one generator of the form $\alpha z^{2}+\beta z w+\gamma w^{2}+\delta(a z+b w)$, which we may assume to be quadric, otherwise $J$ is in I-xii. If $\delta=0$, then $(x, y, z, w)_{\mathfrak{p}}^{2} \subset J_{\mathfrak{p}}$ and we get $(x, y)+(z, w)^{2} \subset J$ since $J$ is p-primary. Both ideals have the same height and multiplicity, and hence are equal. So $J=(x, y)+(z, w)^{2}$, which is impossible since $J: \mathfrak{p}=\left(x, y, z^{2}, z w, w^{2}, a z+b w\right)$. So we assume $\delta=1$. Let $a^{\prime}=a+\alpha z+\beta w$ and $b^{\prime}=b+\gamma w$ we get $\left(x, y, z^{3}, z^{2} w, z w^{2}, w^{3}, a z+b w\right) \subset J$. The ideal on the left is unmixed by Lemma A.12 whenever $\operatorname{ht}(x, y, z, w, a, b)=6$. Since both ideals have the same height and multiplicity then they are equal. Our primary ideal falls into I-iv. If ht $(x, y, z, w, a, b)=5$ then $J$ contains the linear form $z+w$, and $J$ will have the form of I-i after a linear change of variables.

Suppose $x \in J$ and $y \notin J$, then $\left(x, y^{2}, y z, y w, z^{3}, z^{2} w, z w^{2}, w^{3}, z(a z+b w), w(a z+b w)\right) \subset J$. Since the multiplicity of the ideal on the left is 5 , then there exists at least 2 additional generators of the form $\alpha y+\beta z^{2}+\gamma w z+\theta w^{2}+\delta(a z+b w), c y+d z^{2}+e w z+f w^{2}$ with $\operatorname{deg} c=\operatorname{deg} \alpha=1$. The remaining coefficients are in $k$ otherwise we will be in case I-xii. Suppose $\delta=0$ for all such quadrics, then since there are at most three of them in $J$, we get $g y \in J$ for some $g \notin(x, y, z, w)$ which implies $y \in J$ impossible. Hence, we may assume that $\delta=1$. After the following linear change of variables $\left(a^{\prime}=a+\beta z+\gamma w\right.$ and $\left.b^{\prime}=b+\theta w\right)$, we can consider $\beta=\gamma=\theta=0$. After a further linear change of variables, the second quadric has either one of the two following forms $c y+z^{2}$ or $c y+z w$ since any polynomial with two variables over an algebraically closed field splits. Suppose the second quadric is $c y+z^{2}$ then $\left(x, y^{2}, y z, y w, z^{3}, z^{2} w, z w^{2}, w^{3}, \alpha y+a z+b w, c y+z^{2}\right) \subset J$. Note that ht $(x, y, z, w, a, b)=6$. If $\operatorname{ht}(x, y, z, w, b, c)=6$, then by Lemma A.13, we get $J=\left(x, y^{2}, y z, y w, z^{3}, z^{2} w, z w^{2}, w^{3}, \alpha y+a z+b w, c y+z^{2}\right)$ which is case II-vii. When $\operatorname{ht}(x, y, z, w, b, c)=5$, then we may assume either $c=0$ or $b=c$. If $c=0$, then $(y, z, w)^{2}+(x, \alpha y+a z+b w) \subset J$. Since the ideal on the left is unmixed by lemma A.4 of multiplicity 3 then 
it is equal to $J$, which is case I-v. If $b=c$ then $a b y-b z w \in J$ with $b \notin \mathfrak{p}$, then $a y-z w \in J$. By lemma A.15 $J=\left(x, y^{2}, y z, y w, z^{3}, z^{2} w, z w^{2}, w^{3}, \alpha y+a z+b w, b y+z^{2}, a y-z w\right)$, which is I-viii.

Similarly, if the second quadric is $c y+z w$, then $\left(x, y^{2}, y z, y w, z^{3}, z^{2} w, z w^{2}, w^{3}, \alpha y+a z+b w, c y+z w\right) \subset J$. If $\operatorname{ht}(x, y, z, w, a, b, c)=7$, then by lemma A.14, both ideals are equal and this falls in case I-ix. Otherwise, we may assume that $c=a, c=b$ or $c=0$. If $c=a$ or $c=b$ then by lemma A.15, $J$ has the form of I-x or I-xi . If $c=0$, then $z^{2}$ and $w^{2} \in J$, and $J$ has the form of I-v by lemma A.4.

If $x, y \notin J$ then $\left(x^{2}, x y, x z, x w, y^{2}, y z, y w, z^{3}, z^{2} w, z w^{2}, w^{3}, z(a z+b w), w(a z+b w)\right) \subset J$. The multiplicity of the ideal on the left is 6 , so there exists at least three generators of the form $c x+d y+a_{1} z^{2}+a_{2} w z+a_{3} w^{2}+\delta(a z+$ $b w), e x+f y+\alpha_{1} z^{2}+\alpha_{2} w z+b_{3} w^{2}$ and $g x+h y+c_{1} z^{2}+c_{2} w z+c_{3} w^{2}$. We may assume that $c, d, e, f, g, h \in S_{1}$ and the rest of the coefficients are in $k$, or else we get to case II-xi. We may also assume that $\delta \neq 0$ since otherwise when $\delta=0$ for all such quadrics, then $\left(x, y, z^{2}, z w, w^{2}\right)_{\mathfrak{p}} \subset J_{\mathfrak{p}}$. We get $\left(x, y, z^{2}, z w, w^{2}\right) \subset J$ since $J$ is $\mathfrak{p}$-primary, which is a contradiction. Take $\delta=1$. After the following linear change of variables: $a^{\prime}=a+a_{1} z+a_{2} w$ and $b^{\prime}=b+a_{3} w$, we may assume that $a_{1}=a_{2}=a_{3}=0$. After a further linear change of variables, we may assume that the last quadric has the form of either $g x+h y+w z$ or $g x+h y+z^{2}$. Suppose the last quadric is $g x+h y+w z$. Hence $\left(x^{2}, x y, x z, x w, y^{2}, y z, y w, z^{3}, z^{2} w, z w^{2}, w^{3}, c x+d y+a z+b w, e x+f y+\alpha z^{2}+\right.$ $\left.\alpha_{2} w^{2}, g x+h y+z w\right) \subset J$. Further, we may assume that $\alpha_{1}$ or $\alpha_{2} \neq 0$, or else the multiplicity of the ideal on the

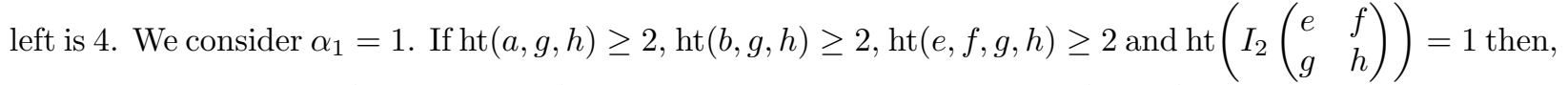
by Lemma A.16, $J=\left(x^{2}, x y, x z, x w, y^{2}, y z, y w, c x+d y+a z+b w, e x+f y+z^{2}+\alpha w^{2}, g x+h y+z w\right)$ with $\alpha \in k$. This is case II-iv. If $I_{2}\left(\begin{array}{ll}e & f \\ g & h\end{array}\right)=0$, then either $J$ is degenerate or we get back to case II-i. If ht $(e, f, g, h)=1$, then the only case to consider is whenever $f=g=0$ and $h=e$, or else $J$ is degenerate. By Lemma A.17, if $\operatorname{ht}(a, b, e)=3$ then $J=\left(x^{2}, x y, x z, x w, y^{2}, y z, y w, c x+d y+a z+b w, e x+z^{2}+\alpha_{2} w^{2}, e y+z w, \alpha_{2} a^{2} y+a b x+b^{2} y\right)$ or $J=\left(x^{2}, x y, x z, x w, y^{2}, y z, y w, c x+d y+a z+b w, e x+z^{2}+\alpha_{2} w^{2}, e y+z w, \alpha_{2} a^{2} y+a b x+b^{2} y\right)$ when $\alpha=0$. This puts us in case II-v or II-vi when $\alpha=0$. Both cases ht $(a, g, h)=1$ or ht $(b, g, h)=1$ are equivalent by a linear change of variables. Suppose $\operatorname{ht}(a, g, h)=1$ and $g=h=a$. By a linear change of variables, we may assume $g=a$ and $h=0$. Hence, by Lemma A.18, $J=\left(x^{2}, x y, x z, x w, y^{2}, y z, y w, c x+d y+a z+b w, e x+\right.$ $\left.f y+\alpha_{1} z^{2}+\alpha_{2} w^{2}, a x+z w, b x-z^{2}\right)$ with ht $(e, f)=2$ or else $J$ is degenerate . This puts us in case II-vii. Suppose the second quadric is $g x+h y+z^{2}$, then $\left(x^{2}, x y, x z, x w, y^{2}, y z, y w, z^{3}, z^{2} w, z w^{2}, w^{3}, c x+d y+a z+\right.$ $\left.b w, e x+f y+\beta z w+\alpha w^{2}, g x+h y+z^{2}\right) \subset J$. We may assume $\beta=0$ or else we get back to the first case by a linear change of variables. If $\alpha=0$ then $J=\left(x^{2}, x y, x z, x w, y^{2}, y z, y w, c x+d y+a z+b w, e x+f y, g x+h y+z^{2}\right)$ when ht $(e, f)=2$ by Lemma A.19. This is case II-viii. Suppose $\alpha \neq 0$. If $\operatorname{ht}(a, e, f) \geq 2, \operatorname{ht}(b, g, h) \geq 2$ and $h t\left(I_{2}\left(\begin{array}{ll}e & f \\ g & h\end{array}\right)\right)$, then $J=\left(x^{2}, x y, x z, x w, y^{2}, y z, y w, c x+d y+a z+b w, e x+f y+w^{2}, g x+h y+z^{2}\right)$ by Lemma A.20. This puts us in case II-ix. If $I_{2}\left(\begin{array}{ll}e & f \\ g & h\end{array}\right)=0$, then either $J$ is degenerate or we we get back to case II-i. If $\operatorname{ht}(a, e, f)=1$ (or equivalently $\mathrm{ht}(b, g, h)=1$ by a change of variables), then by Lemma A.21, $J=\left(x^{2}, x y, x z, x w, y^{2}, y z, y w, c x+d y+a z+b w, a x+w^{2}, g x+h y+z^{2}, b x-z w\right)$ with ht $(b, g, h) \geq 2$. This is case II-x. If $\mathrm{ht}(a, e, f)=\mathrm{ht}(b, g, h)=1$, then we get back to case II-xii.

Case III. $J: P=(y, z, w)^{2}+(x, b y+c z+d w, e y+f z+g w)$.

In this case, we have $(x, y, z, w)(y, z, w)^{2}+(x, y, z, w)(x, b y+c z+d w, e y+f z+g w)$ with $\mathrm{ht}\left(x, y, z, w, I_{2}\left(\begin{array}{lll}b & c & d \\ e & f & g\end{array}\right)\right) \geq 6$. If $J$ contains $x$ then $(x)+(y, z, w)^{3}+(y, z, w)(b y+c z+d w, e y+f z+g w) \subset J$ and the study of this case was done in case III of [11, Proposition 4.4]. This puts us in I-xii. Suppose $x \notin J$ then the above ideal on the left has multiplicity 6 . The Hilbert function of the ideal on the left localized at $\mathfrak{p}$ is $(1,4,1)$. So $J$ contains at least two quadrics which, after a linear change of variables, we may consider to be $a x+b y+c z+d w, h x+e y+f z+g w$. Hence $(y, z, w)^{3}+\left(x^{2}, x y, x z, x w\right)+(a x+b y+c z+d w, h x+e y+f z+g w) \subset J$ and the multiplicity of the ideal on the left is 4 . If $J$ contains one more quadric of the above form then the multiplicity will be 2 . On the other hand, we have $(y, z, w)(b y+c z+d w, e y+f z+g w) \subset J$ which leads 
to $(x, y, z, w)_{\mathfrak{p}}^{2} \subset J_{\mathfrak{p}}$. Since $J$ is $\mathfrak{p}$-primary, then $(x, y, z, w)^{2}+(a x+b y+c z+d w, h x+e y+f z+g w) \subset J$. If $\operatorname{ht}\left(I_{2}\left(\begin{array}{llll}a & b & c & d \\ h & e & f & g\end{array}\right)\right) \geq 2$ then by lemma A.5 both ideals are equal which puts us in case II-i. If $\operatorname{ht}\left(I_{2}\left(\begin{array}{llll}a & b & c & d \\ h & e & f & g\end{array}\right)\right)=1$, then by lemma 3.1 either $J$ is degenerate or the quadrics in $J$ are expressed by at most 8 variables which puts us in case II-xii.

Case IV. $J: P=(x, y, z, w)^{2}+(a x+b y+c z+d w, e x+f y+g z+h w, k x+l y+m z+n w)$. We have $(x, y, z, w)^{3}+(x, y, z, w)(a x+b y+c z+d w, e x+f y+g z+h w, k x+l y+m z+n w) \subset J$ with $\operatorname{ht}\left(x, y, z, w, I_{3}(M)\right) \geq 6$, where $M=\left(\begin{array}{cccc}a & b & c & d \\ e & f & g & h \\ k & l & m & n\end{array}\right)$. Since the multiplicity of the ideal on the left is 6 , then there exist at least three additional quadrics, which after a linear change of variables, we can take to be of the form $a x+b y+c z+d w+q, e x+f y+g z+h w+q^{\prime}$ and $k x+l y+m z+n w+q ", q, q^{\prime}, q " \in$ $(x, y, z, w)^{2}$. After relabeling $a . h, k . . n$, we can assume $q, q^{\prime}, q^{\prime \prime}=0$, without changing ht $\left(x, y, z, w, I_{3}(M)\right)$. Hence $(x, y, z, w)^{3}+(a x+b y+c z+d w, e x+f y+g z+h w, k x+l y+m z+n w) \subset J$. If ht $\left(I_{3}(N)\right) \geq 3$ where

$$
N=\left(\begin{array}{cccccccccccc}
0 & 0 & 0 & 0 & a & b & c & d & e & f & g & h \\
-a & -b & -c & -d & 0 & 0 & 0 & 0 & k & l & m & n \\
-e & -f & -g & -h & -k & -l & -m & -n & 0 & 0 & 0 & 0
\end{array}\right)
$$

then the ideal on the left is unmixed of multiplicity three and hence equal to $J$ by lemma A.25. Suppose $\mathrm{ht}\left(I_{3}((N)) \leq 2\right.$. We have $2 \leq \mathrm{ht}\left(I_{3}(M)\right) \leq \mathrm{ht}\left(I_{2}(M)\right) \bmod (x, y, z, w)$. If ht $\left(I_{2}(M)\right) \geq 3$, then there exists at least three minors $\Delta_{1}, \Delta_{2}$ and $\Delta_{3}$ such that $\left(\Delta_{1}, \Delta_{2}, \Delta_{3}\right)$ is of height three. We may assume $\Delta_{1}=(a f-b e)$. We get $a \Delta_{1}, b \Delta_{1}, e \Delta_{1}$ and $f \Delta_{1} \in I_{3}(N)$ and hence $\Delta_{1}^{2} \in I_{3}(N)$, similarly for $\Delta_{2}, \Delta_{3}$. Hence, we obtain $\operatorname{ht}\left(I_{3}(N)\right) \geq 3$. So the only remaining case to study is whenever $\operatorname{ht}\left(I_{3}(M)\right)=\operatorname{ht}\left(I_{2}(M)\right)=2$. By lemma 4.2, $M$ takes four different forms. If $M$ is of the form 4.2-i then $a\left(x z-y^{2}\right)=z(a x+b y)-y(a y+b z) \in J$, and hence $J=(x, y, z, w)^{3}+\left(a x+b y, a y+b z, x z-y^{2}, k x+l y+m z+n w\right)$ by lemma A.23 which is case II-xi. If $M$ is of the form 4.2-ii then $b(z y-x w)=z(a x+b y)-x(a z+b w) \in J$, and hence $J=(x, y, z, w)^{3}+(a x+b y, a y+b z, z y-x w, k x+l y+m z+n w)$ by lemma A.23 which is case II-xi. If $M$ has the form of 4.2-iv, then by Remark 4.3 and Lemmas A.37, A.38, A.39, we are in case II-xii. If $M$ has the form of 4.2-iii, then we only study one case since all other cases are treated in the same way. We study the case when $\alpha_{2}=\beta_{1}=\beta_{2}=0$ in the ideal $(x, y, z, w)^{3}+\left(a x+\left(\alpha_{1} f+\alpha_{2} g\right) y+\left(\beta_{1} f+\beta_{2} g\right) z, e x+f y+g z, k x+f z+g w\right)$. We get $f\left(z^{2} a-y w a+y w e-y z k\right)=(w e-k z)(a x+f y)-w a(e x+f y+g z)+a z(k x+f z+g w) \in J$ and hence $J=(x, y, z, w)^{3}+\left(a x+f y, e x+f y+g z, k x+f z+g w, z^{2} a-y w a+y w e-y z k\right)$ by lemma A.24. Similarly, in the second form of the matrix we also study the case when $\alpha_{2}=\beta_{1}=\beta_{2}=0$ in the ideal $(x, y, z, w)^{3}+\left(a x+\left(\alpha_{1} f+\alpha_{2} g\right) y+\left(\beta_{1} f+\beta_{2} g\right) z, e x+f y+g z, k x+g z+f w\right)$. We get $J=(x, y, z, w)^{3}+(a x+g y+f z, e x+f y+g z, k x+f z+g w, y a-w a-y e+y k)$ by lemma A.24. All other cases for $\alpha_{i}$ and $\beta_{j}$ are identical and we will omit the details. These cases put us in II-xi.

Case V. $J: P=(x, y, z, w)^{2}+(a x+b y, e x+f y+n z, k x+l y+n w,(a f-b e) w-(a l-k b) z)$.

We have $(x, y, z, w)^{3}+(x, y, z, w)(a x+b y, e x+f y+n z, k x+l y+n w,(a f-b e) w-(a l-k b) z) \subset J$. Since the multiplicity of the ideal on the left is 6 , then there exit at least three additional genrators which we may assume to be of the following $g_{1}=a x+b y+q_{1}, g_{2}=e x+f y+n z+q_{2}, g_{3}=k x+l y+n w+q_{3}$ and $g_{4}=(a f-b e) w-(a l-k b) z+q_{4}$ with $q_{1}, q_{2}, q_{3} \in(z, w)^{2}$ and $q_{4} \in(x, y)^{2}$ after relabeling the variables. The two possibilities for $H_{(R / J)_{P}}$ are either $(1,1,1)$ or $(1,2,0)$. In the first case, we have $q_{i}=0$ for all $i=1 \ldots 4$. It is also easy to show that if three of the $g_{i}^{\prime} s \in J$, then the fourth one is also in $J$. Hence, $(x, y, z, w)^{3}+\left(g_{1}, g_{2}, g_{3}, g_{4}\right) \subset J$. When $\operatorname{ht}\left(I_{5}\left(N^{\prime}\right)\right) \geq 3 \bmod (x, y, z, w)$, with $N^{\prime}$ the matrix defined in lemma A.25, the ideal on the left is unmixed and equal to $J$ by lemma A.25. This puts us in case II-xi. Since $n^{5},(a, b, e, f, k, l)\left((a l-b k)^{3},(a f-b e)^{3},(e l-f k)^{3}\right) \subset I_{5}\left(N^{\prime}\right)$, then $\operatorname{ht}\left(I_{5}\left(N^{\prime}\right)\right) \geq 3$ whenever $\mathrm{ht}\left(I_{2}\left(\begin{array}{ll}a & b \\ e & f \\ k & l\end{array}\right)\right)=2$. Hence, we need to study the case when ht $\left(I_{2}\left(\begin{array}{ll}a & b \\ e & f \\ k & l\end{array}\right)\right)=1$. By [11, Lemma 4.1] 
ON THE PROJECTIVE DIMENSION OF 5 QUADRIC ALMOST COMPLETE INTERSECTIONS WITH LOW MULTIPLICITIE\$7

we have three cases:

Case 1. The matrix has the form: $\left(\begin{array}{ll}a & b \\ e & 0 \\ k & 0\end{array}\right)$. If $\operatorname{ht}(x, y, z, w, a, b, n, e, k) \geq 8$ then by lemma A.26, $J=$ $(x, y, z, w)^{3}+(a x+b y, e x+n z, k x+n w, e w-k z)$ which puts us in case II-xi. If ht $(x, y, z, w, a, b, n, e, k)=7$, we get back to case II-xii.

Case 2. The matrix has the form: $\left(\begin{array}{ll}a & b \\ e & f \\ 0 & 0\end{array}\right)$. Hence by lemma A.27, $J=(x, y, z)^{3}+(w, a x+b y, e x+f y+n z)$ whenever $\operatorname{ht}(x, y, z, w, a, b, n, e, f) \geq 7$, which puts us again in cases II-xi or II-xii.

Case 3. The matrix has the form: $\left(\begin{array}{ll}a & b \\ e & 0 \\ 0 & e\end{array}\right)$. Since there are only three variables in the matrix then we are in case II-xii.

When $H_{(R / J)_{P}}=(1,2,0), J$ contains a quadrics $g_{i}$ with $q_{i} \neq 0$. Since $J$ contains three quadrics, then $(x, y, z, w) g_{l} \in J$ where $g_{l}$ is the $4^{t h}$ quadric. If $\operatorname{ht}\left(I_{5}\left(N^{\prime}\right)\right) \geq 3$, then $J=(x, y, z, w)^{3}+\left(g_{i}, g_{j}, g_{k}\right)+$ $(x, y, z, w) g_{l}$ for $i \neq j \neq k \neq l$, by lemma A.28 which puts us in case II-xi. Hence, we study the case when $\mathrm{ht}\left(I_{2}\left(\begin{array}{ll}a & b \\ e & f \\ k & l\end{array}\right)\right)=1$. As above, this puts us in II-xi or II-xii.

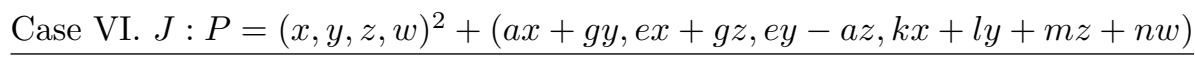

This case is similar to Case III. Since we have $(x, y, z, w)^{3}+(x, y, z, w)(a x+g y, e x+g z, e y-a z, k x+l y+$ $m z+n w) \subset J$ and of multiplicity 6 , then there exist at least three additional quadrics. We may assume these quadrics to be three of the following $g_{1}=a x+g y+q_{1}, g_{2}=e x+g z+q_{2}, g_{3}=e y-a z+q_{3}$ and $g_{4}=k x+l y+m z+n w+q_{4}$ with $q_{1} \in(z, w)^{2}, q_{2} \in(y, w)^{2}, q_{3} \in(x, w)^{2}$ and $q_{4}=0$ after relabeling the variables. When $H_{(R / J)_{P}}=(1,1,1)$, then $q_{1}=q_{2}=q_{3}=0$. Hence, by lemma A.29, $J=(x, y, z, w)^{3}+(a x+$ $g y, e x+g z, e y-a z, k x+l y+m z+n w)$ if ht $(x, y, z, w, a, g, e, k, l, m, n) \geq 8$, which puts us in case II-xi. If $\mathrm{ht}(x, y, z, w, a, g, e, k, l, m, n) \leq 7$, then we are back to case II-xii.

When $H_{(R / J)_{P}}=(1,2,0)$, there exists an $i$ such that $q_{i} \neq 0$. In that case, by lemma A.30,J=(x,y,z,w) ${ }^{3}+$ $\left(g_{i}, g_{j}, k x+l y+m z+n w\right)+(x, y, z, w) g_{l}$ for $i, j, l=1, \ldots 3$, which are cases II-xi or II-xii.

\section{The Projective Dimension of 5 QUAdriC Almost COMPlete intersections}

In this section, we let $I$ be an almost complete intersection generated by 5 quadrics. In [13, Question 6.2] and [13, Question 10.2], the authors asked the following question:

Question 5.1. Let $S$ be a polynomial ring and let $I$ be an ideal of $S$ generated by $n$ quadrics, having $h t(I)=h$. Is it true that $p d(S / I) \leq h(n-h+1)$ ?

We give an affirmative answer to their question for almost complete intersections generated by 5 quadrics with small multiplicities. We show that, when $I$ has multiplicity $\leq 3$, then $\operatorname{pd}(S / I) \leq 8$. When the multiplicity is less or equal than 2 , we make use of the inequality between the multiplicity and the CohenMacaulay defect, found in [3, Theorem 2.5]. If the multiplicity is three, then $I^{\text {un }}$ is one of the following types: $\langle 3 ; 1\rangle,\langle 1 ; 3\rangle,\langle 1,2 ; 1,1\rangle,\langle 1,1 ; 1,2\rangle$ and $\langle 1,1,1 ; 1,1,1\rangle$. We prove in every case, that $\operatorname{pd}(S / I) \leq 8$.

Notation 5.2. In this section, we use the following notation:

- $S$ is a polynomial ring over an algebraically closed field $k$,

- $I=\left(q_{1}, q_{2}, q_{3}, q_{4}, q_{5}\right)$ where $q_{i}$ are polynomials of degree 2 and height $(I)=4$

- $L=\left(q_{1}, q_{2}, q_{3}, q_{4}\right): I=\left(q_{1}, q_{2}, q_{3}, q_{4}\right): I^{u n}$

Corollary 5.3. Let $I$ be an almost complete intersection generated by 5 quadrics with $e(S / I) \leq 2$, then $p d(S / I) \leq 6$ 
Proof. If $e(S / I) \leq 2$, then by [3, Theorem 2.5], $e(S / I) \geq \operatorname{dim}(S / I)-\operatorname{depth}(S / I)$. Hence $2 \geq e(S / I) \geq$ $\operatorname{pd}(S / I)-4$, which implies the assertion.

Before we move to multiplicity three, we need the following lemmas.

Lemma 5.4. If $I^{\text {un }}$ or $L=\left(q_{1}, q_{2}, q_{3}, q_{4}\right): I$ contains two linear forms then $p d(S / I) \leq 6$

Proof. The proof goes along the same line as the proof of Lemma 3.4 in [11]. We suppose $x$ and $y$ are two linear forms contained in $I^{u n}$. Since $I \subset(I, x, y) \subset I^{u n}$, then $\operatorname{ht}(I, x)=4$. Hence, after relabeling the quadrics, we may assume $x, y, q_{1}, q_{2}$ form a regular sequence. Let $L^{\prime}=\left(x, y, q_{1}, q_{2}\right): I=\left(x, y, q_{1}, q_{2}\right): I^{u n}$. Since $L^{\prime}$ contains a complete intersection of two linear forms and two quadrics then $e\left(S / L^{\prime}\right) \leq 4$. If $e\left(S / L^{\prime}\right)=4$ then $L^{\prime}=\left(x, y, q_{1}, q_{2}\right)$ and $L^{\prime}$ is $\mathrm{CM}$, hence $\operatorname{pd}(S / I) \leq 5$ by Lemma 2.5. If $e\left(S / L^{\prime}\right)=1$ then $L$ is CM by theorem 2.6 and $\operatorname{pd}(S / I) \leq 5$ again by Lemma 2.5. If $e\left(S / L^{\prime}\right)=2$, then write $L^{\prime}=(x, y)+L^{\prime \prime}$, where $x$ and $y$ are regular on $L^{\prime \prime}, L^{\prime \prime}$ unmixed of multiplicity $e\left(S / L^{\prime \prime}\right)=2$ and height $h t\left(L^{\prime \prime}\right)=2$. By [6, Proposition 11], $\operatorname{pd}\left(S / L^{\prime \prime}\right) \leq 3$ which yields to $\operatorname{pd}\left(S / L^{\prime}\right) \leq 5$ and hence $\operatorname{pd}(S / I) \leq 6$. Finally if $e\left(S / L^{\prime}\right)=3$ consider $J=\left(x, y, q_{1}, q_{2}\right): L^{\prime}$, by Theorem $2.3, e(S / J)=e\left(S /\left(x, y, q_{1}, q_{2}\right)\right)-e\left(S / L^{\prime}\right)=4-3=1$. So $J$ is $\mathrm{CM}$ by Theorem 2.6. Hence $L^{\prime}$ is $\mathrm{CM}$ and $\operatorname{pd}\left(S / L^{\prime}\right)=3$, which implies that $\operatorname{pd}(S / I) \leq 4$.

Lemma 5.5. Suppose $I \subset K \cap J$ where $K=K^{\prime}+(q), h t(K)=h t(J)=4, h t\left(K^{\prime}\right)=3$ and $q$ a quadric. Then there exists a quadric $q^{\prime} \in K \cap J$ such that $K=K^{\prime}+\left(q^{\prime}\right)$.

Proof. For each $i=1 \cdots 5$, write $q_{i}=f_{i}+\alpha_{i} q$ where $f_{i} \in K^{\prime}$ and $\alpha_{i} \in k$. If $\alpha_{i}=0$ for all $i$, one has $I \subset K^{\prime}$ which implies ht $\left(K^{\prime}\right) \geq 4$ and gives a contradiction. Hence, one may assume $\alpha_{i} \neq 0$ for some $i$. Take $q^{\prime}=q_{i}$.

In the next theorems, we suppose $e(S / I)=3$.

Theorem 5.6. If $I^{\text {un }}$ is of type $\langle 3 ; 1\rangle$, then $p d(S / I) \leq 8$.

Proof. If $I^{u n}$ is of type $\langle 3 ; 1\rangle$, then it contains a linear form say $x$ by Proposition 2.7 . We write $I^{u n}=(x)+I^{\prime}$, where $I^{\prime}$ is unmixed of type $\langle 3 ; 1\rangle$ and height three. By [11, Theorem 2.10], $I^{\prime}$ contains another linear form. Hence $\operatorname{pd}(S / I) \leq 5$ by Lemma 5.4 .

Theorem 5.7. If $I^{\text {un }}$ is of type $\langle 1 ; 3\rangle$, then $p d(S / I) \leq 8$.

Proof. If $I^{u n}$ is of type $\langle 1 ; 3\rangle$, then $I^{u n}$ is one of proposition 4.4. When $I^{u n}$ contains two linear forms then by lemma $5.4, \operatorname{pd}(S / I) \leq 6$. This cover cases I- $(i)$ to $(i v)$. In cases I- $(v)$ to $(x i)$, I- $(x i i i)$ and II- $(x i i)$, all quadrics in $I^{u n}$ are expressible in terms of at most 8 variables. Hence by lemma $2.10, \operatorname{pd}(S / I) \leq 8$. When the quadrics of $I^{u n}$ generate an ideal of height at most three, we get a contradiction $\operatorname{since}$ ht $(I)=4$. In the cases II- $(i)$ to $(x)$, we note by Lemmas A.5, A.10, A.11, A.16, A.17, A.19 and A.20, that any ideal $L$ directly linked to $I^{\text {un }}$ satisfies $\operatorname{pd}(S / L) \leq 6$. Also by Lemmas A.18 and A.21, we get $\operatorname{pd}\left(\operatorname{Corker}\left(\partial_{5}^{*}\right)\right) \leq 6$ where $\partial_{i}$ is the $i^{\text {th }}$ differential map in the resolution of $I^{u n}$. Hence $\operatorname{pd}(S / I) \leq 7$ by Lemma 2.5 and Proposition 2.11 .

Theorem 5.8. If $I^{u n}$ is of type $\langle 1,2 ; 1,1\rangle$, then $\operatorname{pd}(S / I) \leq 6$.

Proof. If $I^{u n}=(x, y, z, w) \cap(u, v, s, q)$ where $q \in S_{2}$ and the rest in $S_{1}$. We may assume that $q \in(x, y, z, w)$ by Lemma 5.5, say $q=a x+b y+c z+d w$. If ht $(x, y, z, w, u, v, s) \leq 5$, then $I^{u n}$ contains two linear forms and $\operatorname{pd}(S / I) \leq 5$ by Lemma 5.4. If ht $(x, y, z, w, u, v, s)=6$ then $I^{u n}$ contains a linear form say $x$. We write $I^{u n}=(x)+(y, z, w) \cap(v, s, q)$, with $q=b y+c z+d w$. This composition is the sum of a linear form and a height three unmixed ideal of type $\langle 1,2 ; 1,1\rangle$. By the proof of [11, Lemma 7.2], either $(y, z, w) \cap(v, s, q)$ contains a linear form or $\operatorname{pd}\left(\operatorname{Corker}\left(\partial_{4}^{*}\right)\right) \leq 5$ where $\partial_{i}$ is the $i^{\text {th }}$ differential map in the resolution of $(y, z, w) \cap(v, s, q)$. In the first case we get $\operatorname{pd}(S / I) \leq 5$ by Lemma 5.4 , and in the second case $\operatorname{pd}\left(\operatorname{Corker}\left(\partial_{5}^{*}\right)\right) \leq 6$ where $\partial_{i}$ is the $i^{\text {th }}$ differential map in the resolution of $I^{\text {un }}$. Hence $\operatorname{pd}(S / I) \leq 6$ by Proposition 2.11 . We may assume that $\operatorname{ht}(x, y, z, w, u, v, s)=7$. We have $I^{u n}=(q, x u, x v, x s, y u, y v, y s, z u, z v, z s, w u, w v, w s)$, and we let $L=(x u, y v, s z, q): I^{u n}$. By Lemma A.31, $\operatorname{pd}(S / L) \leq 5$, and hence $\operatorname{pd}(S / I) \leq 6$ by Lemma 2.5 . 
ON THE PROJECTIVE DIMENSION OF 5 QUADRIC ALMOST COMPLETE INTERSECTIONS WITH LOW MULTIPLICITIE\$9

Theorem 5.9. If $I^{u n}$ is of type $\langle 1,1 ; 1,2\rangle$, then $p d(S / I) \leq 8$.

Proof. Suppose that $I^{u n}$ is $(u, v, s, t) \cap L_{2}$ where $L_{2}$ is as Proposition 3.4.

- $\underline{\text { Case } 1}$. If $L_{2}=\left(x, y, z, w^{2}\right)$, then $I^{u n}$ can be expressed by at most 8 variables, and pd $(S / I) \leq 8$ by Lemma 2.10 .

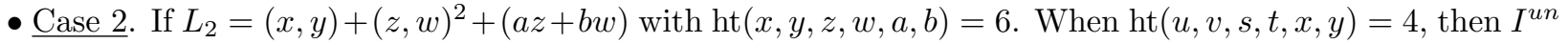
contains two linear forms and $\operatorname{pd}(S / I) \leq 6$ by Lemma 5.4. If $\operatorname{ht}(u, v, s, t, x, y)=5$, then we can write $I^{u n}=$ $(x)+(v, s, t) \cap\left((y)+(z, w)^{2}+(a z+b w)\right)$ with ht $(v, s, t, y)=4$. The case of $(v, s, t) \cap\left((y)+(z, w)^{2}+(a z+b w)\right)$ was studied in the proof of in [11, Lemma 7.3]. We get either the quadrics of $(v, s, t) \cap\left((y)+(z, w)^{2}+(a z+b w)\right)$ generate a height two ideal, or they are expressed with at most 6 variables. Hence, the quadrics of $I^{u n}$ generate a height three ideal, or they are expressed with at most 7 variables. So $\operatorname{pd}(S / I) \leq 7$. We then assume that $\operatorname{ht}(u, v, s, t, x, y)=6$. We may assume that the degree of $a$ and $b$ is one and $a z+b w \in(u, v, s, t) \cap L_{2}$, or else the quadrics of $I^{u n}$ will be expressed with at most 8 variables. Hence, if $h t(u, v, s, t, z, w)=6$ then $(u, v, s, t) \cap L_{2}=(a z+b w)+(u, v, s, t)\left(x, y, z^{2}, z w, w^{2}\right)$, and all quadrics of $I^{u n}$ generate an ideal of height at most three. This contradicts our assumption about $I$. If $\operatorname{ht}(u, v, s, t, z, w) \leq 5$, then we may assume that $z=u$. Hence $a u+b w \in(u, v, s, t) \cap L_{2}$. We must have $w \in(v, s, t)$ or $b \in(u, v, s, t)$. Hence $\operatorname{ht}(x, y, z, w, u, v, s, t, a, b) \leq 8$, and all quadrics in $I^{u n}$ can be expressed with at most 8 variables. So $\operatorname{pd}(S / I) \leq 8$.

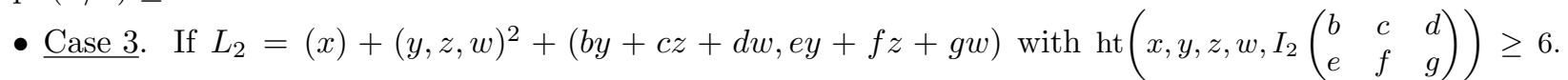
We may assume that the degree of $b, c, d, e, f, g$ is 1 , and $b y+c z+d w, e y+f z+g w \in(u, v, s, t) \cap L_{2}$, or else the quadrics of $I^{u n}$ will be expressed with at most 8 variables. If ht $(x, y, z, w, u, v, s, t)=8$, then $(u, v, s, t) \cap L_{2}=(b y+c z+d w, e y+f z+g w)+(u, v, s, t)\left(x, y^{2}, y z, y w, z^{2}, z w, w^{2}\right)$, and the quadrics of $I^{u n}$ generate an ideal of height three. If $\operatorname{ht}(x, y, z, w, u, v, s, t) \leq 7$ and $x \in(u, v, s, t)($ we take $x=u)$, then we may write $I^{u n}=(x)+(b y+c z+d w, e y+f z+g w)+(v, s, t) \cap(y, z, w)^{2}$. By the proof of $[11$, Lemma 7.3], we get that all quadrics of $I^{u n}$ are expressed with at most 7 variables. We suppose next that $\operatorname{ht}(x, y, z, w, u, v, s, t) \leq 7$ and $x \notin(u, v, s, t)$. Suppose first that ht $(x, y, z, w, u, v, s, t)=7$ and $y=u$. We let $I=\left(f_{1} l_{1}, f_{2} l_{2}, f_{3} l_{3}, b y+c z+d w, e y+f z+g w\right)$ with $e(S / I)=3$, where $f_{i}=\alpha_{i} x+\beta_{i} y, \alpha_{i}, \beta_{i} \in k$ and $l_{i}$ are linear forms. But $I+(x, y)=(x, y, c z+d w, f z+g w)$ and ht $\left(x, y, I_{2}\left(\begin{array}{lll}b & c & d \\ e & f & g\end{array}\right)\right)=4$. Hence $\operatorname{ht}(S /(I+(x, y)))=4$ and $e(S /(I+(x, y)))=4$. This is impossible, since $I \subset(I+(x, y))$ and $e(S / I)=3$. If $\operatorname{ht}(x, y, z, w, u, v, s, t)=6$, then we take $y=u$ and $z=v$. We have $b y+c z+d w, e y+f z+g w \in(u, v, s, t)$. Hence $d \in(s)$ and $g=0$. The case $d=g=0$ cannot happen due to the height restriction on the matrix of minors $I_{2}$. So, $I^{u n}=\left(x y, x z, x s, x t, y^{2}, y z, y w, z^{2}, z w, w^{2} t, w^{2} s, b y+c z+w s, e y+f z\right)$. Since $\operatorname{ht}\left(x, y, z, w, s, t, I_{2}\left(\begin{array}{lll}b & c & d \\ e & f & g\end{array}\right)\right) \geq 7$ then, by Lemma A.32, $\operatorname{pd}(S / L)=5$ where $L$ is directly linked to $I^{u n}$. This implies that $\operatorname{pd}(S / I) \leq 6$ by Lemma 2.5. If $\operatorname{ht}(x, y, z, w, u, v, s, t)=5$, then we may assume further that $w=s$. Hence $I^{u n}=\left(x t, x y, x z, x w, y^{2}, y z, y w, z^{2}, z w, w^{2}, b y+c z+d w, e y+f z+g w\right)$. This case can be treated in a similar manner as whenever ht $(x, y, z, w, u, v, s, t)=7$. Finally, if ht $(x, y, z, w, u, v, s, t)=4$, then $x \in(u, v, s, t)$, and this case is already treated above.

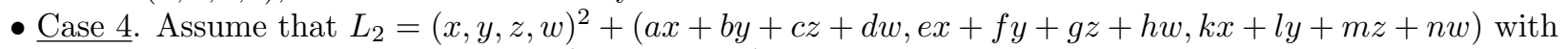
$\operatorname{ht}\left(x, y, z, w, I_{3}(M)\right) \geq 6$, where $M=\left(\begin{array}{cccc}a & b & c & d \\ e & f & g & h \\ k & l & m & n\end{array}\right)$. We use a similar argument as the previous cases. If $\operatorname{ht}(x, y, z, w, u, v, s, t)=8$, then the quadrics of $I^{u n}$ generate a height at most three. If ht $(x, y, z, w, u, v, s, t)=$ 6 or 7 , then both cases will be treated the same way as whenever ht $(x, y, z, w, u, v, s, t)=7$ in Case 3 . If $\operatorname{ht}(x, y, z, w, u, v, s, t)=5$, then we may assume that $x=u, y=v$ and $z=s$. Since at least two of $a x+b y+c z+d w, e x+f y+g z+h w, k x+l y+m z+n w \in(x, y, z, t)$, then we may assume that $d=t$ and $h=0$ after a linear change of variables. We get either $(u, v, s, t) \cap L_{2}=(a x+b y+c z+t w, e x+f y+g z, k x+l y+m z)+$ $(x, y, z, t) \cap(x, y, z, w)^{2}$, or $(a x+b y+c z+t w, e x+f y+g z)+(x, y, z, t) \cap\left[(x, y, z, w)^{2}+(k x+l y+m z+n w)\right]$. In the first case, since $\operatorname{ht}\left(x, y, z, w, I_{3}(M)\right)=6$, we get ht $\left(\begin{array}{ccc}e & f & g \\ k & l & m\end{array}\right) \geq 2 \bmod (x, y, z, w)$. By Lemma 
A.33 we obtain $\operatorname{pd}(S / L)=5$ for an ideal $L$ directly linked to $I^{u n}$. Hence, $\operatorname{pd}(S / I) \leq 6$ by Lemma 2.5 . In the second case, we suppose $n \notin t$ or else we are back to the first case. Since ht $\left(I_{3}(m)\right) \geq 2 \bmod$ $(x, y, z, w)$ then, by Lemma A.34, $\operatorname{pd}(S / L)=5$ for an ideal $L$ directly linked to $I^{u n}$. This implies that $\operatorname{pd}(S / I) \leq 6$ by Lemma 2.5. If $\operatorname{ht}(x, y, z, w, u, v, s, t)=4$, then $x=u, y=v, z=s$ and $w=t$. $I^{u n}=$ $(a x+b y+c z+t w, e x+f y+g z, k x+l y+m z)+(x, y, z, w)^{2}$ which is p-primary. Hence by Proposition 5.7, $\operatorname{pd}(S / I) \leq 7$.

- Case 5. If $L_{2}=(x, y, z, w)^{2}+(a x+b y, e x+f y+n z, k x+l y+n w,(a f-b e) w-(a l-k b) z)$ with ht $(a, b, n)=3$, then in most cases the quadrics of $I^{u n}$ generate an ideal of height at most three, or we can use the same method as whenever ht $(x, y, z, w, u, v, s, t)=7$ of Case 3 and show a contradiction. The only remaining cases to study is whenever $(u, v, s, t) \cap L_{2}=(a x+b y, k x+l y+n w)+(x, y, z, t) \cap\left((x, y, z, w)^{2}, e x+f y+n z\right)$ or $(a x+b y, k x+l y+m z+n w)+(x, y, s, w) \cap\left((x, y, z, w)^{2}, e x+f y+n z\right)$. Both cases will be treated the same way, so we will prove only one of them. Suppose $I^{u n}=(a x+b y, k x+l y+n w)+(x, y, z, t) \cap\left((x, y, z, w)^{2}, e x+f y+n z\right)=$ $(x, y, z)^{2}+\left(w x, w y, w z, w^{2} t, a x+b y, k x+l y+n w, e x+f y+n z\right)$, then by Lemma A.35, $\operatorname{pd}(S / L)=5$ for an ideal $L$ directly linked to $I^{u n}$. Hence $\operatorname{pd}(S / I) \leq 6$.

- Case 6. Finally, let $L_{2}=(x, y, z, w)^{2}+(a x+g y, e x+g z, e y-a z, k x+l y+m z+n w)$. We may suppose that $k x+l y+m z+n w \in(u, v, s, t)$ or else the quadrics of $I^{u n}$ will be expressed in terms of at most 8 variables. If $\mathrm{ht}(x, y, z, w, u, v, s, t)=8$, then the quadrics of $I^{u n}$ can be expressed with at most 8 variables. If $\operatorname{ht}(x, y, z, w, u, v, s, t)=7$, then we may assume that $x=u$. This case will be solved the same way as whenever $\mathrm{ht}(x, y, z, w, u, v, s, t)=7$ of Case 3. If $\mathrm{ht}(x, y, z, w, u, v, s, t)=6$, then we may assume that $x=u$ and $y=v$. In this case, the quadrics of $I^{u n}$ will generate an ideal of at most three which is a contradiction. If $\operatorname{ht}(x, y, z, w, u, v, s, t)=5$, then we may assume that $x=u, y=v$, and $z=s$ or $w=t$. The only cases to study are $I^{u n}=(a x+g y, e x+g z, e y-a z, k x+l y+m z+n w)+(x, y, z, t) \cap(x, y, z, w)^{2}$ or $I^{u n}=(a x+g y, e x+g z, e y-a z, k x+l y+m z+n w)+(x, y, s, w) \cap(x, y, z, w)^{2}$. Both cases will be treated the same way, so we prove the first one. In the first case, $I^{u n}=(a x+g y, e x+g z, e y-a z, k x+l y+m z+$ $\left.n w, x^{2}, x y, x z, x w, y^{2}, y z, y w, z^{2}, z w, w^{2} t\right)$. By Lemma A.36, $\operatorname{pd}(S / L) \leq 5$ for an ideal $L$ linked to $I^{u n}$. Hence $\operatorname{pd}(S / I) \leq 6$.

Theorem 5.10. If $I^{u n}$ is of type $\langle 1,1,1 ; 1,1,1\rangle$, then $p d(S / I) \leq 6$.

Proof. Write $I^{u n}=\cap_{i=1}^{3} L_{i}$ where $L_{i}=\left(x_{i}, y_{i}, z_{i}, w_{i}\right)$. If $\operatorname{ht}\left(L_{i}+L_{j}\right)=8$ for any $i \neq j$, then all quadrics of $J$ will be expressed with at most 8 variables which are $x_{i}, y_{i}, z_{i}, w_{i}, x_{j}, y_{j}, z_{j}, w_{j}$. Otherwise $\operatorname{ht}\left(L_{i}+L_{j}\right) \leq 7$ for any $i \neq j$. In that case, either all generators are expressed with at most 8 variables or $I^{u n}$ contains a linear form. In the latter case, we write $I^{u n}=(x)+I^{\prime}$ where $I^{\prime}=\cap_{i=1}^{3} L_{i}^{\prime}$ and $L_{i}^{\prime}=\left(y_{i}, z_{i}, w_{i}\right)$. Hence by the proof of [11, Lemma 7.4], either $I^{\prime}$ contains a linear form or the quadrics of $I^{\prime}$ are expressed with at most 6 variables or all generators are expressed with at most 6 variables. So either $I^{u n}$ contains two linear forms or the quadrics of $I^{u n}$ are expressed with at most 7 variables or the generators of $I^{u n}$ are expressed with at most 8 variables. Hence, pd $(S / I) \leq 8$ by Lemma 5.4 or Corollary 2.10 .

\section{Appendix: Resolution of Primary Ideals}

We follow the same techniques as of [11, Appendix A]. We resolve the unmixed primary ideals generically and check the exactness of the resolution by using the Buchsbaum-Eisenbud exactness criteria. For that, if $\mathbb{F}$ is the resolution of $S / I$ and $\partial_{i}$ denotes the $i^{t h}$ differential map, then it suffices to check that $\operatorname{ht}\left(I_{r_{j}}\left(\partial_{j}\right)\right) \geq j$ for all $j$, where $r_{j}=\sum_{i=j}^{p}(-1)^{p-i} \operatorname{rank}\left(F_{i}\right)$ and $I_{r}\left(\partial_{j}\right)$ is the $r \times r$ minors of the matrix associated to the $j^{\text {th }}$ differential. Further if $\operatorname{ht}\left(I_{r_{j}}\left(\partial_{j}\right)\right) \geq j+1$ for $j>\operatorname{ht}(I)$ then the ideal is unmixed by [12, Proposition 2.4]. In the case when $\mathrm{ht}(I)=4$, it suffices to show

$$
\operatorname{ht}\left(I_{r_{j}}\left(\partial_{j}\right)\right) \geq\left\{\begin{array}{ccc}
j & \text { for } & j=1,2,3,4 \\
j+1 & \text { for } & j \geq 5
\end{array}\right.
$$

We give a complete proof for lemma A.1, the rest is done in a similar manner. The computations were done by using the computer algebra Macaulay 2 [9]. 
ON THE PROJECTIVE DIMENSION OF 5 QUADRIC ALMOST COMPLETE INTERSECTIONS WITH LOW MULTIPLICITIES1

Lemma A.1. If

$$
J=(x, y, z, w)^{2}+(a x+b y+c z+d w, e x+f y+g z+h w, k x+l y+m z+n w)
$$

where ht $\left(x, y, z, w, I_{3}\left(\begin{array}{cccc}a & b & c & d \\ e & f & g & h \\ k & l & m & n\end{array}\right)\right)=6$ then $J$ is $(x, y, z, w)-\operatorname{primary}, p d(S / J)=5$ and $e(S / J)=2$.

Proof. First, we let $N=(x, y, z, w)^{2}$ with a minimal free resolution obtained by using the Eagon- Northcott complex

$$
0 \rightarrow S^{4} \stackrel{D_{4}}{\longrightarrow} S^{15} \stackrel{D_{3}}{\longrightarrow} S^{20} \stackrel{D_{2}}{\longrightarrow} S^{10} \stackrel{D_{1}}{\longrightarrow} S \rightarrow S / N \rightarrow 0
$$

We then consider the complex

$$
0 \rightarrow R^{3} \stackrel{\partial_{5}}{\longrightarrow} R^{16} \stackrel{\partial_{4}}{\longrightarrow} R^{33} \stackrel{\partial_{3}}{\longrightarrow} R^{32} \stackrel{\partial_{2}}{\longrightarrow} R^{13} \stackrel{\partial_{1}}{\longrightarrow} R
$$

with $\partial_{1}=\left(a x+b y+c z+d w, e x+f y+g z+h w, k x+l y+m z+n w, D_{1}\right)$

$$
\partial_{2}=\left(\begin{array}{ccccccccccccc} 
& -a & 0 & 0 & 0 & -e & 0 & 0 & 0 & -k & 0 & 0 & 0 \\
& -b & -a & 0 & 0 & -f & -e & 0 & 0 & -l & -k & 0 & 0 \\
& 0 & -b & 0 & 0 & 0 & -f & 0 & 0 & 0 & -l & 0 & 0 \\
D_{2} & 0 & 0 & -a & 0 & -g & 0 & -e & 0 & -m & 0 & -k & 0 \\
& 0 & -c & -b & 0 & 0 & -g & -f & 0 & 0 & -m & -l & 0 \\
& 0 & -c & 0 & 0 & 0 & -g & 0 & 0 & 0 & -m & 0 \\
& -d & 0 & 0 & -a & -h & 0 & 0 & -e & -n & 0 & 0 & -k \\
& 0 & -d & 0 & -b & 0 & -h & 0 & -f & 0 & -n & 0 & -l \\
& 0 & 0 & -d & -c & 0 & 0 & -h & -g & 0 & 0 & -n & -m \\
& 0 & 0 & 0 & -d & 0 & 0 & 0 & -h & 0 & 0 & 0 & -n \\
0 \ldots 0 & x & y & z & w & 0 & 0 & 0 & 0 & 0 & 0 & 0 & 0 \\
0 \ldots 0 & 0 & 0 & 0 & 0 & x & y & z & w & 0 & 0 & 0 & 0 \\
0 \ldots 0 & 0 & 0 & 0 & 0 & 0 & 0 & 0 & 0 & x & y & z & w
\end{array}\right)
$$

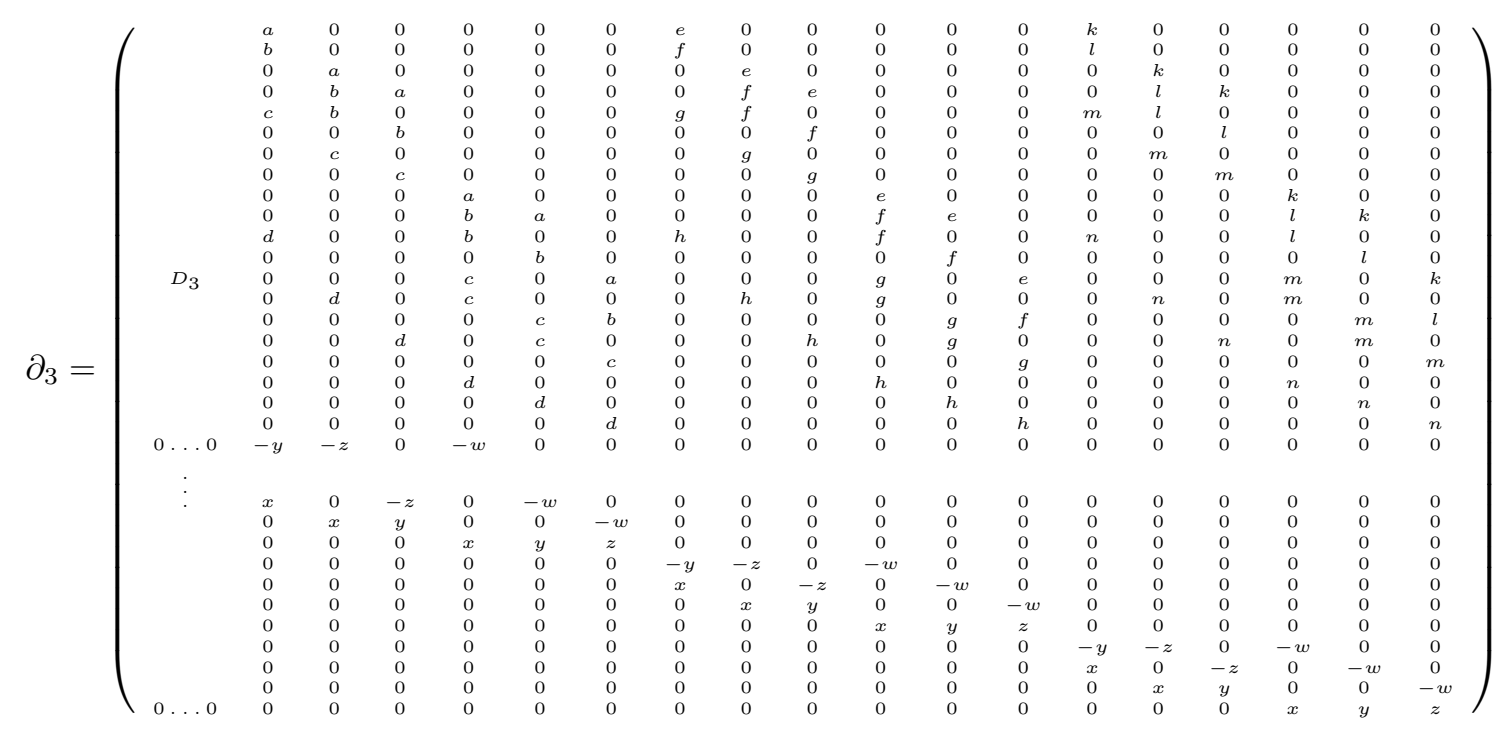


and $\partial_{5}=\left(\begin{array}{ccc}a & e & k \\ b & f & l \\ c & g & m \\ d & h & n \\ -w & 0 & 0 \\ z & 0 & 0 \\ -y & 0 & 0 \\ x & 0 & 0 \\ 0 & -w & 0 \\ 0 & z & 0 \\ 0 & -y & 0 \\ 0 & x & 0 \\ 0 & 0 & -w \\ 0 & 0 & z \\ 0 & 0 & -y \\ 0 & 0 & x\end{array}\right)$

We note that $x^{2} \in I_{1}\left(\partial_{1}\right)$ and $x^{12}, y^{12} \in I_{12}\left(\partial_{2}\right)$. Further we note that $x^{20}, y^{20}$ and $z^{20} \in I_{20}\left(\partial_{3}\right)$ and $x^{13}, y^{13}, z^{13}$ and $w^{13} \in I_{13}\left(\partial_{4}\right)$. Finally $x^{3}, y^{3}, z^{3}, w^{3}$ and $I_{3}\left(\begin{array}{cccc}a & b & c & d \\ e & f & g & h \\ k & l & m & n\end{array}\right) \in I_{3}\left(\partial_{5}\right)$.

Since ht $\left(I_{3}\left(\begin{array}{cccc}a & b & c & d \\ e & f & g & h \\ k & l & m & n\end{array}\right)\right) \geq 2 \bmod (x, y, z, w)$ then the complex is exact and resolves $J$. So $J$ is unmixed with $\operatorname{pd}(S / J)=5$. Further $\lambda\left(S_{\mathfrak{p}} / J_{\mathfrak{p}}\right)=2$ and $\sqrt{J}=(x, y, z, w)$. Hence, $J$ is $(x, y, z, w)$-primary with multiplicity $e(S / J)=2$.

Lemma A.2. If

$$
J=(x, y, z, w)^{2}+(a x+b y, e x+f y+n z, k x+l y+n w,(a l-b k) z-(a f-b e) w)
$$

where $h t\left(x, y, z, w, n, I_{2}\left(\begin{array}{ll}a & b \\ e & f \\ k & l\end{array}\right)\right)=7$, then $J$ is $(x, y, z, w)$-primary with $p d(S / J)=6$ and $e(S / J)=2$.

Lemma A.3. If

$$
J=(x, y, z, w)^{2}+(a x+b y, e x+b z, e y-a z, k x+l y+m z+n w)
$$

with $h t(x, y, z, w, a, b, e)=8$, then $J$ is $(x, y, z, w)$ - primary, $p d(S / J)=6$ and $e(S / J)=2$.

Lemma A.4. If

$$
J=(y, z, w)^{2}+(x, b y+c z+d w)
$$


ON THE PROJECTIVE DIMENSION OF 5 QUADRIC ALMOST COMPLETE INTERSECTIONS WITH LOW MULTIPLICITIES3 with $h t(x, y, z, w, b, c, d) \geq 6$, then $J$ is $(x, y, z, w)$-primary, $p d(S / J)=5$ and $e(S / J)=3$.

Lemma A.5. If

$J=(x, y, z, w)^{2}+(a x+b y+c z+d w, e x+f y+g z+h w)$
with $h t\left(I_{2}\left(\begin{array}{llll}a & b & c & d \\ e & f & g & h\end{array}\right)\right) \geq 2 \bmod (x, y, z, w)$, then $J$ is $(x, y, z, w)$-primary, pd $(S / J)=5$ and $e(S / J)=3$.

Furthermore

$L=\left(x^{2}, y^{2}, z^{2}, w^{2}\right): J=\left(x^{2}, y^{2}, z^{2}, w^{2}, x y z y w, x(y z(b g-c f)-y w(b h-d f)+z w(c h-d g), y(x z(a g-c e)-\right.$ $x w(a h-d e)+z w(c h-d g), z(x y(a f-b e)-x w(a h-d e)+y w(b h-f d)), w(x y(a f-b e)-x z(a g-c e)+y z(b g-c f))$ and $\operatorname{pd}(S / L)=6$.

Lemma A.6. If

$$
J=(x, y, z, w)^{2}+(a x+b y, e x+b z, e y-a z)
$$

with $h t(x, y, z, w, a, b, e)=7$, then $J$ is $(x, y, z, w)$-primary, $p d(S / J)=6$ and $e(S / J)=3$.

Lemma A.7. If

$$
J=\left(x, y, z^{2}, w z, w^{3}, w^{2}+a z\right)
$$

then $J$ is $(x, y, z, w)$-primary with $p d(S / J)=4$ and $e(S / J)=3$.

Lemma A.8. If

$$
J=(x)+(y, z)^{2}+\left(w y, w z, w^{2}, b y+c z+d w\right)
$$

with $h t(x, y, z, w, b, c, d) \geq 6$ then $J$ is $(x, y, z, w)$-primary with $p d(S / J)=5$ and $e(S / J)=3$.

Lemma A.9. If

$$
J=(x)+(y, z)^{2}+\left(w y, w z, w^{2}+b y+c z, e y+f z\right)
$$

with $h t(x, y, z, w, e, f)=6$ then $J$ is $(x, y, z, w)$-primary with $p d(S / J)=5$ and $e(S / J)=3$.

Lemma A.10. If

$$
J=(x, y, z)^{2}+\left(w x, w y, w z, w^{2}+a x+b y+c z, e x+f y+g z, k x+l y+m z\right)
$$

with ht $\left(I_{2}\left(\begin{array}{ccc}e & f & g \\ k & l & m\end{array}\right)\right) \geq 2 \bmod (x, y, z, w)$, then $J$ is $(x, y, z, w)$-primary with $p d(S / J)=5$ and $e(S / J)=$ 3.

Furthermore

$L=\left(x^{2}, y^{2}, z^{2}, w^{2}+a x+b y+c z\right): J=\left(x^{2}, y^{2}, z^{2}, w^{2}+a x+b y+c z, x y z, w(x y(e l-k f)-x z(e m-g k)+\right.$ $y z(f m-g l))$ and $p d(S / L)=5$

Lemma A.11. If

$$
J=(x, y, z)^{2}+\left(w x, w y, w z, w^{2}+a x+b y+c z, e x+f y, k x+f z, k y-e z\right)
$$

with $h t(x, y, z, w, e, f, k)=7$, then $J$ is $(x, y, z, w)$-primary with $p d(S / J)=6$ and $e(S / J)=3$.

Furthermore,

$L=\left(x^{2}, y^{2}, z^{2}, w^{2}+a x+b y+c z\right): J=\left(x^{2}, y^{2}, z^{2}, w^{2}+a x+b y+c z, x y z, x y w k+x z w e-y z w f\right)$ and $p d(S / L)=5$

Lemma A.12. If

$$
J=(x, y)+(z, w)^{3}+(a z+b w)
$$

with $h t(x, y, z, w, a, b)=6$, then $J$ is $(x, y, z, w)$-primary with $p d(S / J)=5$ and $e(S / J)=3$.

Lemma A.13. If

$$
J=(x)+y(y, z, w,)+(z, w)^{3}+\left(a y+b z+c w, d y+z^{2}\right)
$$

with $h t(x, y, z, w, b, c)=h t(x, y, z, w, c, d)=6$, then $J$ is $(x, y, z, w)$-primary with $p d(S / J)=5$ and $e(S / J)=$ 3. 
Lemma A.14. If

$$
J=(x)+y(y, z, w,)+(z, w)^{3}+(a y+b z+c w, d y+z w)
$$

with $h t(x, y, z, w, b, c, d)=7$, then $J$ is $(x, y, z, w)$-primary with $p d(S / J)=5$ and $e(S / J)=3$.

Lemma A.15. If

$$
J=(x)+y(y, z, w)+(z, w)^{3}+\left(a y+b z+c w, c y+z^{2}, b y-z w\right)
$$

or

or

$$
J=(x)+y(y, z, w)+(z, w)^{3}+\left(a y+b z+c w, c y+z w, b y-w^{2}\right)
$$

$$
J=(x)+y(y, z, w)+(z, w)^{3}+\left(a y+b z+c w, b y+z w, c y-z^{2}\right)
$$

with $h t(y, z, w, b, c)=5$, then $J$ is $(x, y, z, w)$-primary with $p d(S / J)=5$ and $e(S / J)=3$.

Lemma A.16. If

$$
J=(x, y)^{2}+(x, y)(z, w)+\left(a x+b y+c z+d w, e x+f y+z^{2}+\alpha w^{2}, g x+h y+z w\right)
$$

with $h t(x, y, z, w, c, d)=6, h t(c, g, h) \geq 2, h t(d, g, h) \geq 2, h t(e, f, g, h) \geq 2$ and $h t\left(I_{2}\left(\begin{array}{ll}e & f \\ g & h\end{array}\right)\right)=1$, then $J$ is $(x, y, z, w)$-primary with $p d(S / J)=5$ and $e(S / J)=3$.

Lemma A.17. If

$$
J=(x, y)^{2}+(x, y)(z, w)+\left(a x+b y+c z+d w, e x+z^{2}+w^{2}, e y+z w, c^{2} y+c d x+d^{2} y\right)
$$

or

$$
J=\left(x^{2}, x y, x z, x w, y^{2}, y z, y w, w^{3}, a x+b y+c z+d w, e x+z^{2}, e y+z w, c x+d y\right)
$$

with $h t(c, d, e)=3 \bmod (x, y, z, w)$, then $J$ is $(x, y, z, w)$-primary with $p d(S / J)=5$ and $e(S / J)=3$.

Lemma A.18. If

$$
J=(x, y)^{2}+(x, y)(z, w)+\left(a x+b y+c z+d w, e x+f y+z^{2}+\alpha w^{2}, c x+z w, d x-z^{2}\right)
$$

with $h t(c, d)=h t(e, f)=2 \bmod (x, y, z, w)$, then $J$ is $(x, y, z, w)$-primary with $p d(S / J)=5$ and $e(S / J)=3$.

Lemma A.19. If

$$
J=(x, y)^{2}+(x, y)(z, w)+\left(a x+b y+c z+d w, e x+f y, g x+h y+z^{2}\right)
$$

with $h t(c, d)=h t(e, f)=2 \bmod (x, y, z, w)$, then $J$ is $(x, y, z, w)$-primary with $p d(S / J)=5$ and $e(S / J)=3$.

Lemma A.20. If

$$
J=(x, y)^{2}+(x, y)(z, w)+\left(a x+b y+c z+d w, e x+f y+w^{2}, g x+h y+z^{2}\right)
$$

with $h t(x, y, z, w, c, d)=6, h t(c, e, f) \geq 2, h t(d, g, h) \geq 2$ and $h t\left(I_{2}\left(\begin{array}{ll}e & f \\ g & h\end{array}\right)\right)=1$, then $J$ is $(x, y, z, w)$ primary with $p d(S / J)=5$ and $e(S / J)=3$.

Lemma A.21. If

$$
J=(x, y)^{2}+(x, y)(z, w)+\left(a x+b y+c z+d w, c x+w^{2}, g x+h y+z^{2}, d x-z w\right)
$$

with $h t(x, y, z, w, c, d)=6$, ht $(d, g, h) \geq 2$, then $J$ is $(x, y, z, w)$-primary with $p d(S / J)=5$ and $e(S / J)=3$.

Lemma A.22. If

$$
J=(x, y, z, w)^{3}+(a x+b y+c z+d w, e x+f y+g z+h w, k x+l y+m z+n w)
$$

with $h t\left(x, y, z, w, I_{3}(N)\right) \geq 7$ where

$$
N=\left(\begin{array}{cccccccccccc}
0 & 0 & 0 & 0 & a & b & c & d & e & f & g & h \\
-a & -b & -c & -d & 0 & 0 & 0 & 0 & k & l & m & n \\
-e & -f & -g & -h & -k & -l & -m & -n & 0 & 0 & 0 & 0
\end{array}\right)
$$

then $J$ is $(x, y, z, w)$-primary with $p d(S / J)=6$ and $e(S / J)=3$. 
ON THE PROJECTIVE DIMENSION OF 5 QUADRIC ALMOST COMPLETE INTERSECTIONS WITH LOW MULTIPLICITIE85

Lemma A.23. If

$$
J=(x, y, z, w)^{3}+\left(a x+b y, a y+b z, x z-y^{2}, k x+l y+m z+n w\right)
$$

or

$$
J=(x, y, z, w)^{3}+(a x+b y, a y+b z, y z-x w, k x+l y+m z+n w)
$$

with $h t(x, y, z, w, a, b, k, l, m, n) \geq 7$, then $J$ is $(x, y, z, w)$-primary with $p d(S / J)=6$ and $e(S / J)=3$.

Lemma A.24. If

$$
J=(x, y, z, w)^{3}+\left(a x+f y, e x+f y+g z, k x+l y+m z+n w, z^{2} a-y w a+y w e-y z k\right)
$$

or

$$
J=(x, y, z, w)^{3}+(a x+g y+f z, e x+f y+g z, k x+f z+g w, y a-w a-y e+y k)
$$

with $h t(x, y, z, w, a, e, f, g, k) \geq 8$, then $J$ is $(x, y, z, w)$-primary with $p d(S / J)=7$ and $e(S / J)=3$.

Lemma A.25. If

$$
\left.J=(x, y, z, w)^{3}+(a x+b y, e x+f y+n z, k x+l y+n w,(a f-b e) w-(a l-k b) z)\right)
$$

with $h t(x, y, z, w, a, b, n)=7$ and $h t\left(I_{5}(N)\right) \geq 3 \bmod (x, y, z, w)$ where

$$
N^{\prime}=\left(\begin{array}{cccccccccccccc}
0 & 0 & 0 & 0 & a & b & 0 & 0 & e & f & n & 0 & 0 & 0 \\
-a & -b & 0 & 0 & 0 & 0 & 0 & 0 & k & l & 0 & n & 0 & 0 \\
-e & -f & -n & 0 & -k & -l & 0 & -n & 0 & 0 & 0 & 0 & 0 & 0 \\
0 & 0 & a f-b e & 0 & 0 & 0 & a l-b k & 0 & 0 & 0 & e l-f k & 0 & n & 0 \\
0 & 0 & 0 & a f-b e & 0 & 0 & 0 & a l-b k & 0 & 0 & 0 & e l-f k & 0 & n
\end{array}\right),
$$

then $J$ is $(x, y, z, w)$-primary with $p d(S / J)=6$ and $e(S / J)=3$.

Lemma A.26. If

$$
J=(x, y, z, w)^{3}+(a x+b y, e x+n z, k x+n w, e w+k z)
$$

with $h t(x, y, z, w, a, b, n, e, k) \geq 8$, then $J$ is $(x, y, z, w)$-primary with $p d(S / J)=7$ and $e(S / J)=3$.

Lemma A.27. If

$$
J=(x, y, z)^{3}+(w, a x+b y, e x+f y+n z)
$$

with $h t(x, y, z, w, a, b, n, e, f) \geq 7$, then $J$ is $(x, y, z, w)$-primary with $p d(S / J)=6$ and $e(S / J)=3$.

Lemma A.28. If

$$
J=(x, y, z, w)^{3}+\left(g_{i}, g_{j}, g_{k}\right)+(x, y, z, w) g_{l}
$$

where $g_{1}=a x+b y+q_{1}, g_{2}=e x+f y+n z+q_{2}, g_{3}=k x+l y+n w+q_{3}$, and $\left.g_{4}=(a f-b e) w-(a l-k b) z\right)+q_{4}$, with $q_{1}, q_{2}, q_{3} \in(z, w)^{2}, q_{4} \in(x, y)^{2}$ and one of the $q_{i} \neq 0, h t(x, y, z, w, a, b, n)=7$ and $h t\left(I_{5}(N)\right) \geq 3 \bmod$ $(x, y, z, w)$ where

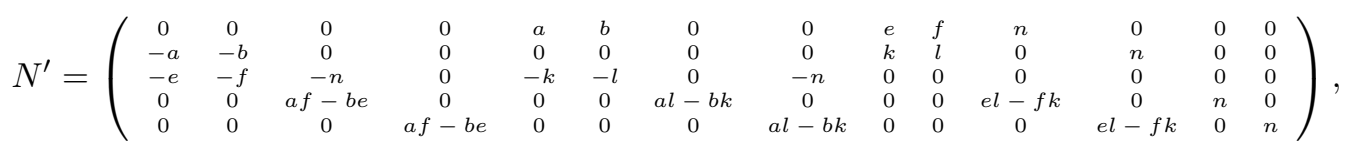

then $J$ is $(x, y, z, w)$-primary with $p d(S / J)=6$ and $e(S / J)=3$.

Lemma A.29. If

$$
J=(x, y, z, w)^{3}+(a x+g y, e x+g z, e y-a z, k x+l y+m z+n w)
$$

with $h t(x, y, z, w, a, g, e, k, l, m, n) \geq 8$ then $J$ is $(x, y, z, w)$-primary with $p d(S / J)=7$ and $e(S / J)=3$.

Lemma A.30. If

$$
J=(x, y, z, w)^{3}+\left(g_{i}, g_{j}, k x+l y+m z+n w\right)+(x, y, z, w) g_{l}
$$

where $g_{1}=a x+g y+q_{1}, g_{2}=e x+g z+q_{2}$ and $g_{3}=e y-a z+q_{3}$ with $q_{1} \in(z, w)^{2}, q_{2} \in(y, w)^{2}, q_{3} \in(x, w)^{2}$, $h t(x, y, z, w, a, e, g, k, l, m, n) \geq 8$ then $J$ is $(x, y, z, w)$-primary with $p d(S / J)=7$ and $e(S / J)=3$. 
Lemma A.31. If

$$
J=(x u, y u, z u, w u, x v, y v, z v, w v, x l, y l, z l, w l, a x+b y+c z+d w)
$$

where $h t(x, y, z, w, u, v, l)=7$ then $J$ is unmixed with $p d(S / J)=6$ and $e(S / J)=3$.

Furthermore,

$$
L=(x u, y v, z l, a x+b y+c z+d w): J=(x u, y v, z l, a x+b y+c z+d w, x y z, d u v l)
$$

and $p d(S / L)=5$.

Lemma A.32. If

$$
J=\left(x y, x z, x s, x t, y^{2}, y z, y w, z^{2}, z w, w^{2} t, w^{2} s, b y+c z+w s, e y+f z\right)
$$

where $h t\left(x, y, z, w, s, t, I_{2}\left(\begin{array}{lll}b & c & d \\ e & f & g\end{array}\right)\right) \geq 7$ then $J$ is unmixed with $p d(S / J)=6$ and $e(S / J)=3$.

Furthermore,

$$
\begin{aligned}
L & =\left(x t, y^{2}, z^{2}, b y+c z+w s\right): J \\
& =\left(x t, y^{2}, z^{2}, b y+c z+w s, y z s t, x y z w, t s^{2}(y e-z f)\right)
\end{aligned}
$$

and $p d(S / L)=5$.

Lemma A.33. If

$$
J=(a x+b y+c z+t w, e x+f y+g z, k x+l y+m z)+(x, y, z, t) \cap(x, y, z, w)^{2}
$$

where $h t\left(x, y, z, w, I_{2}\left(\begin{array}{ccc}e & f & g \\ k & l & m\end{array}\right)\right)=6$, then $J$ is unmixed, $p d(S / J)=5$ and $e(S / J)=3$.

Furthermore,

$$
\begin{aligned}
L & =\left(x^{2}, a x+b y+c z+t w, e x+f y+g z, k x+l y+m z\right): J \\
& =\left(x^{2}, a x+b y+c z+t w, e x+f y+g z, k x+l y+m z, x(g l-f m), t(g l-f m)^{2}\right)
\end{aligned}
$$

and $p d(S / L)=5$.

\section{Lemma A.34. If}

$$
J=(a x+b y+c z+t w, e x+f y+g z)+(x, y, z, t) \cap\left[(x, y, z, w)^{2}+(k x+l y+m z+n w)\right]
$$

where $h t\left(x, y, z, w, t, I_{2}\left(\begin{array}{ccc}e & f & g \\ k & l & m\end{array}\right)\right)=7$ then $J$ is unmixed, $p d(S / J)=5$ and $e(S / J)=3$.

Furthermore,

$$
\begin{aligned}
L & =\left(x^{2}, y^{2}, z^{2}, w^{2} t\right): J \\
& =\left(x^{2}, y^{2}, z^{2}, w^{2} t, x y z y w, x y z t I_{3}\left(\begin{array}{lll}
a & b & c \\
e & f & g \\
k & l & m
\end{array}\right)-x y w t I_{3}\left(\begin{array}{lll}
a & b & t \\
e & f & 0 \\
k & l & n
\end{array}\right)+x z w t I_{3}\left(\begin{array}{lll}
a & c & t \\
e & g & 0 \\
k & m & n
\end{array}\right)-y z w t I_{3}\left(\begin{array}{lll}
b & c & t \\
f & g & 0 \\
l & m & n
\end{array}\right)\right) \\
& \text { and } p d(S / L)=5 .
\end{aligned}
$$

Lemma A.35. If

$$
J=(x, y, z)^{2}+\left(w x, w y, w z, w^{2} t, a x+b y, k x+l y+n w, e x+f y+n z\right)
$$

where $h t(x, y, z, w, a, b, n)=7$ then $J$ is unmixed, $p d(S / J)=5$ and $e(S / J)=3$.

\section{Furthermore,}

$$
L=\left(x^{2}, y^{2}, z^{2}, k x+l y+n w\right): J=\left(x^{2}, y^{2}, z^{2}, k x+l y+n w, x y z n, x y n(a f-b e)+z n^{2}(y b-x z)\right)
$$
and $p d(S / L)=5$. 
ON THE PROJECTIVE DIMENSION OF 5 QUADRIC ALMOST COMPLETE INTERSECTIONS WITH LOW MULTIPLICITIES7

Lemma A.36. If

$$
J=\left(a x+g y, e x+g z, e y-a z, k x+l y+m z+n w, x^{2}, x y, x z, x w, y^{2}, y z, y w, z^{2}, z w, w^{2} t\right)
$$

where $h t(x, y, z, w, a, e, g)=7$ then $J$ is unmixed, $p d(S / J)=6$ and $e(S / J)=3$.

Furthermore,

$$
L=\left(x^{2}, y^{2}, z^{2}, k x+l y+n w\right): J=\left(x^{2}, y^{2}, z^{2}, k x+l y+m z+n w, x y z n, n^{2}(x a z+x y e-g y z)\right)
$$

and $p d(S / L)=5$.

Lemma A.37. If

$$
J=(x, y, z, w,)^{3}+\left(a x+b y, a y+b z, y^{2}-x z, k x+l y+m z+n w\right)
$$

or

$$
J=(x, y, z, w,)^{3}+(a x+b y, a z+b w, y z-x w, k x+l y+m z+n w)
$$

where $n \neq 0$ and $h t(x, y, z, w, a, b, k, l, m, n) \geq 7$ then $J$ is $(x, y, z, w)$-primary, $p d(S / J)=6$ and $e(S / J)=3$. If

$$
J=(x, y, z, w,)^{3}+\left(a x+b y, a y+b z, a z+b w, y^{2}-x z,, z^{2}-y w, y z-x w\right)
$$

and $h t(x, y, z, w, a, b) \geq 6$ then $J$ is $(x, y, z, w)$-primary, $p d(S / J)=5$ and $e(S / J)=3$.

Lemma A.38. If

$$
\begin{gathered}
J=(x, y, z, w)^{3}+\left(d x+a y, c x+b y+a z, b z+a w, y w c+z^{2} d-y w d\right) \text { or } \\
J=(x, y, z, w)^{3}+\left(d x+b y, c x+b y+a z, b z+a w, y z c-z^{2} d+y w d\right) \text { or } \\
J=(x, y, z, w)^{3}+\left(d x+a y, c x+b z+a w, b y+a z, y^{2} c-y z d+z^{2} d-y w d\right) \text { or } \\
J=(x, y, z, w)^{3}+\left(d x+b y, c x+b z+a w, b y+a z, y z c-z^{2} d+y w d\right) \text { or } \\
J=(x, y, z, w)^{3}+(a x+b y, c x+b y+a z, d x+b z+a w, x z c-y w c-x y d+y z d) \text { or } \\
J=(x, y, z, w)^{3}+\left(a x+b y, c x+b y+a z, d x+b z+a w, x z c-z w c-x y d+z^{2} d\right)
\end{gathered}
$$

with $h t(x, y, z, w, a, b, c, d)=8$ then $J$ is $(x, y, z, w)$-primary, $p d(S / J)=7$ and $e(S / J)=3$.

\section{Lemma A.39.}

$$
\begin{gathered}
\text { If } J=(x, y, z, w)^{3}+(d x+a y, c x+b y+a z, b z+a w, y w c+y z d-z w d) \text { or } \\
J=(x, y, z, w)^{3}+(d x+a y+a z, c x+b y+a z, a z+b w, y w c+z w c+y z d-z w d) \text { or } \\
J=(x, y, z, w)^{3}+\left(d x+a y+b z, c x+b y+a z, a z+b w, z^{2} c-y w c-y z d+z w d\right) \text { or } \\
\quad J=(x, y, z, w)^{3}+\left(d x+b y, c x+b z+a w, a y+b z, y^{2} c-y z d+z w d\right) \text { or } \\
J=(x, y, z, w)^{3}+\left(d x+b y+b z, c x+b z+a w, a y+b z, y^{2} c+y z c-y z d+z w d\right) \text { or } \\
J=(x, y, z, w)^{3}+\left(d x+b y+a z, c x+b z+a w, a y+b z, y^{2} c-z^{2} c-y z d+z w d\right) \text { or }
\end{gathered}
$$

with $h t(x, y, z, w, a, b, c, d)=8$ then $J$ is $(x, y, z, w)$-primary, $p d(S / J)=7$ and $e(S / J)=3$.

Lemma A.40.

$$
\text { If } J=(x, y, z, w)^{2}+(a x+b y, k z+l w, l y+a z, k x+b w, b z-l x, a w-k y)
$$

with $h t(x, y, z, w, a, b, k, l)=8$ then $J$ is $(x, y, z, w)$-primary, $p d(S / J)=7$ and $e(S / J)=3$.

\section{ACKNOWLEDGMENT}




\section{REFERENCES}

[1] T. Ananyan and M. Hochster, Small Subalgebras of Polynomial Rings and Stillman's Conjecture, preprint: arXiv:1610.09268. 1

[2] T. Ananyan and M. Hochster, Ideals generated by quadratic polynomials, Math.Res. Lett. 19 (2012), 233 - 244. 1, 3

[3] S. H. Hassanzadeh and E. Tavanfar, Annihilators of Koszul Homologies and almost complete intersections, preprint: arxiv.1702.01111. 1, 17, 18

[4] D. Eisenbud, The geometry of syzygies: A second course in commutative algebra and algebraic geometry, Graduate Texts in Mathematics 229, Springer-Verlag, New York (2005). 3, 4

[5] B. Engheta, Bounds on projective dimension, Ph.D. thesis, University of Kansas, (2005). 2

[6] B. Engheta, On the projective dimension and the unmixed part of three cubics, J. Algebra 316 (2007), 715 - 734. 1, 2,18

[7] B. Engheta, A bound on the projective dimension of three cubics, J. Sym. Comp. 45 (2010), 60 - 73.1

[8] G. Flystad, J. McCullough, and I. Peeva, Three themes of syzygies, Bull. Amer. Math. Soc. (N.S.) 53 (2016), no. 3, 415-435. 1

[9] Daniel R. Grayson and Michael E. Stillman, Macaulay 2, http://www.math.uiuc.edu/Macaulay2/ 20

[10] J. Harris, Algebraic Geometry. A first course. graduate Texts in Mathematics 133. Springer-Verlag, New York (1992). 3

[11] C. Huneke, P.Mantero, J. Mccullough, and A. Seceleanu, A tight bound on the projective dimension of four quadrics, to appear in J. Pure Appl. Algebra (2017). 1, 3, 4, 5, 6, 14, 15, 16, 18, 19, 20

[12] C. Huneke, P. Mantero, J. Mccullough, and A. Seceleanu, Multiple structures with arbitrarily large projective dimension supported on linear subspaces, J. Algebra 447 (2016), 183 - 205. 1, 20

[13] C. Huneke, P. Mantero, J. McCullough, and A. Seceleanu, The projective dimension of codimension two algebras presented by quadrics, J. Algebra 393 (2013), 170 - 186. 1, 17

[14] C. Huneke and I. Swanson, Integral closure of ideals, rings, and modules, London Mathematical Society Lecture Note Series, vol. 336, Cambridge University Press, Cambridge, 2006. 2

[15] N. Manolache, Codimension two linear varieties with nilpotent structures, Math. Zeitschrift 210 (1992), no. 4, $573-580.2$

[16] N. Manolache, Cohen-Macaulay nilpotent schemes, Recent Advances in Geometry and Topology, Cluj Univ. Press, Cluj-Napoca (2004), pp. 235 - 248. 2

[17] P. Mantero and J. McCullough, A finite classification of $(x, y)$-primary ideals of low multiplicity, to appear in Collect. Math. 1

[18] P. Mantero and J. McCullough, The projective dimension of three cubics is at most 5, preprint. 1

[19] J. McCullough and A. Seceleanu, Bounding projective dimension, Commutative Algebra, Springer-Verlag London Ltd., London, 2012. 1

[20] M. Nagata, Local rings, Robert E. Kreiger Publishing Co., 1975. 3

[21] I. Peeva and M. Stillman, Open problems on syzygies and Hilbert functions, J. Commut. Algebra 1, (2009), $159-195$. 1

[22] P. Samuel, La notion de multiplicite en algebre et en geometrie algebrique, I and II, J. Math. Pures. Appl. 30, (1951), $159-274.3$

[23] C. Peskine and L. Szpiro, Liaison des varietes algebriques, Invent. Math. 26 (1974), 271 - 302. 2

[24] J. Vatne, Multiple structures and Hartshorne's conjecture, Comm. Algebra, 37 (2009), no. 11, 3861-3873. 2

Department of Mathematics, American University of Beirut, Beirut, Lebanon.

E-mail address: se24@aub.edu.1b 